\title{
Layer-Specific fMRI Responses to Excitatory and Inhibitory Neuronal Activities in the Olfactory Bulb
}

\author{
Alexander John Poplawsky, ${ }^{1 \star}$ Mitsuhiro Fukuda, ${ }^{1 \star}$ Matthew Murphy, ${ }^{2}$ and Seong-Gi Kim ${ }^{1,3,4}$ \\ Departments of ${ }^{1}$ Radiology and ${ }^{2}$ Ophthalmology, University of Pittsburgh, Pittsburgh, Pennsylvania 15203 , ${ }^{3}$ Center for Neuroscience Imaging Research, \\ Institute for Basic Science, Suwon, 440-330, Korea, and ${ }^{4}$ Departments of Biomedical Engineering and Biological Sciences, Sungkyunkwan University, \\ Suwon, 440-330, Korea
}

\begin{abstract}
High-resolution functional magnetic resonance imaging (fMRI) detects localized neuronal activity via the hemodynamic response, but it is unclear whether it accurately identifies neuronal activity specific to individual layers. To address this issue, we preferentially evoked neuronal activity in superficial, middle, and deep layers of the rat olfactory bulb: the glomerular layer by odor (5\% amyl acetate), the external plexiform layer by electrical stimulation of the lateral olfactory tract (LOT), and the granule cell layer by electrical stimulation of the anterior commissure (AC), respectively. Electrophysiology, laser-Doppler flowmetry of cerebral blood flow (CBF), and blood oxygenation level-dependent (BOLD) and cerebral blood volume-weighted (CBV) fMRI at 9.4 T were performed independently. We found that excitation of inhibitory granule cells by stimulating LOT and AC decreased the spontaneous multi-unit activities of excitatory mitral cells and subsequently increased CBF, CBV, and BOLD signals. Odor stimulation also increased the hemodynamic responses. Furthermore, the greatest CBV fMRI responses were discretely separated into the same layers as the evoked neuronal activities for all three stimuli, whereas BOLD was poorly localized with some exception to the poststimulus undershoot. In addition, the temporal dynamics of the fMRI responses varied depending on the stimulation pathway, even within the same layer. These results indicate that the vasculature is regulated within individual layers and CBV fMRI has a higher fidelity to the evoked neuronal activity compared with BOLD. Our findings are significant for understanding the neuronal origin and spatial specificity of hemodynamic responses, especially for the interpretation of laminar-resolution fMRI.
\end{abstract}

Key words: BOLD fMRI; cerebral blood flow; cerebral blood volume fMRI; GABA; hemodynamic response

\section{Significance Statement}

Functional magnetic resonance imaging (fMRI) is a noninvasive, in vivo technique widely used to map function of the entire brain, including deep structures, in animals and humans. However, it measures neuronal activity indirectly by way of the vascular response. It is currently unclear how finely the hemodynamic response is regulated within single cortical layers and whether increased inhibitory neuronal activities affect fMRI signal changes. Both laminar specificity and the neural origins of fMRI are important to interpret functional maps properly, which we investigated by activating discrete rat olfactory bulb circuits.

\section{Introduction}

Functional magnetic resonance imaging (fMRI) is widely used to map brain function in animals and humans by measuring the hemodynamic response to neuronal activity. Although the relationship between these processes has been investigated extensively, it is still questionable whether fMRI is useful for studying

\footnotetext{
Received March 15, 2015; revised Aug. 25, 2015; accepted Sept. 15, 2015.

Author contributions: A.J.P., M.F., and S.-G.K. designed research;A.J.P. and M.F. performed research; A.J.P., M.F., and M.M. analyzed data; A.J.P., M.F., and S.-G.K. wrote the paper.

This work was supported by the National Institutes of Health (Grant NS07391 to A.J.P., Grants NS079143 and EB018903 to M.F., and Grants EB003324 and 1S10RR026503-01 to S.-G.K.) and the Institute for Basic Science (Grant IBS-R015-D1 to S.-G.K.). We thank Nathan Urban of Carnegie Mellon University and Gordon Shepherd of Yale for insightful discussions on the olfactory bulb and review of an early version of this manuscript and Ping Wang, Bistra Iordanova, Alberto Vazquez, and Kristy Hendrich for assisting in our experiments and data analyses.
}

laminar processing because it is unclear if laminar fMRI responses accurately reflect layer-specific neuronal activity. Laminar structures are commonly found throughout the brain, including the cerebral cortex, hippocampus, and olfactory bulb. Each layer processes different information, has unique patterns of inputs and outputs, and has distinctive intrinsic connectivity that are not fully understood in vivo. Despite evidence from studies in layer 4 of the primary sensory cortex, where the largest fMRI

\footnotetext{
The authors declare no competing financial interests.

*A.J.P. and M.F. contributed equally to this work.

Correspondence should be addressed to Seong-Gi Kim, IBS Center for Neuroscience Imaging Research, Sungkyunkwan University, Suwon, 440-330, Korea. E-mail: seonggikim@skku.edu.

DOI:10.1523/JNEUROSCI.1015-15.2015

Copyright $\odot 2015$ the authors $\quad 0270-6474 / 15 / 3515263-13 \$ 15.00 / 0$
} 
A

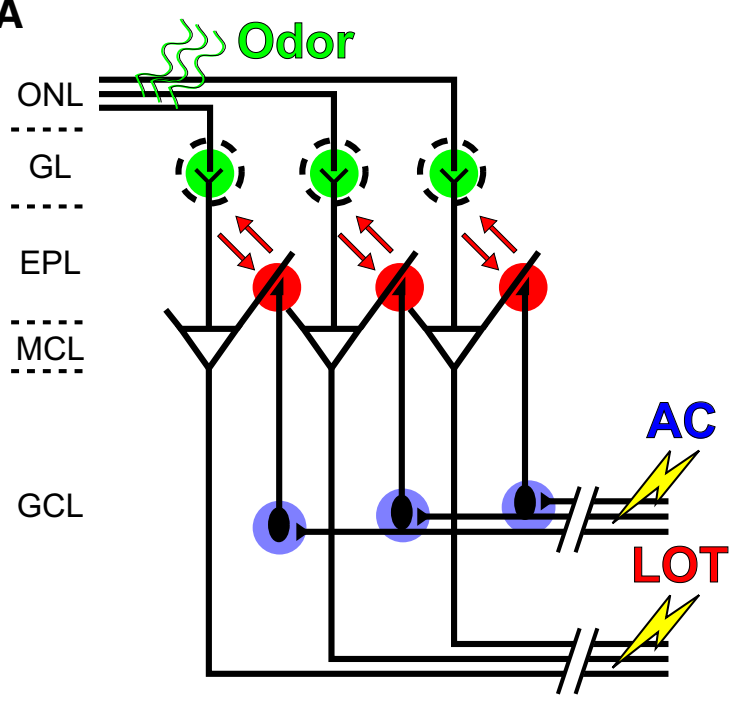

B

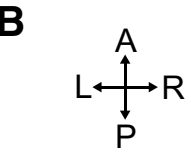

C

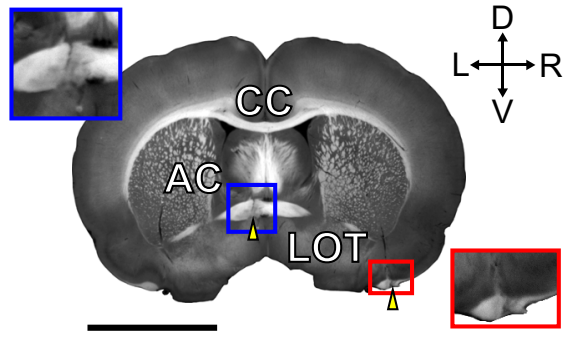

Figure 1. Experimental paradigm. A, Main circuit of the olfactory bulb (OB) where the superficial glomerular layer (GL, green circles), middle external plexiform layer (EPL, red circles), and deep granule cell layer (GCL, blue circles) were preferentially activated by odor ( $5 \%$ amyl acetate in mineral oil) and electrical stimulations ( -150 to $-200 \mu \mathrm{A}, 100-200 \mu \mathrm{s}$ pulse width, $40 \mathrm{~Hz}$ ) of the lateral olfactory tract (LOT) and anterior commissure (AC), respectively. Note that antidromic stimulation of excitatory mitral cells (white bodies) by LOT stimulation evokes synaptic activity in inhibitory granules cells (black bodies) in EPL via reciprocal dendrodendritic synapses (double arrows). B, Stimulating electrodes were stereotaxically positioned to right $L O T$ and left AC and local field potentials (LFPs) were measured in right GCL to optimize the placement of the stimulating electrodes before fMRI. AON, Anterior olfactory nucleus; $A$, anterior; $P$, posterior; R, right; L, left. C, Positions of stimulating electrodes were histologically verified by electrolytic lesions after fMRI. CC, Corpus callosum; D, dorsal; V, ventral.

and neuronal responses are located in the same input layers (Harel et al., 2006; Zhao et al., 2006; Ress et al., 2007; Koopmans et al., 2010; Polimeni et al., 2010; Goense et al., 2012; Chen et al., 2013), the similarity of fMRI activation profiles to the baseline blood volume variation across cortical layers obscures whether fMRI responses are actually neural specific or if they are a simple reflection of the inherent vascular composition. Indeed, hypercapnia produces the greatest fMRI response in cortical layer 4 independent of neural activity, similar to visual stimulation (Yen, 2011). In addition, the laminar circuits of the cortex are highly interconnected and the activity of each layer is functionally dependent on the others. Therefore, although some attempts have been made (Olman et al., 2012), it is difficult to test how the neuronal activity of any single, specific layer contributes to the vascular response using the cortical model, which obscures whether current layer-specific fMRI activation truly reflects laminar processing.

The olfactory bulb circuitry has unique features that are ideal to test whether the vasculature is regulated within layers. Because sensory input, output, and modulatory inputs are clearly organized in different bulb layers (Shepherd, 1972), synapses can be directly and independently activated in single layers (100-200 $\mu \mathrm{m}$ thickness) using different stimuli. In fact, the largest fMRI responses evoked by odor stimulation are found in the glomerular layer (GL; Fig. 1A, green circles; Xu et al., 2000; Xu et al., 2003; Poplawsky and Kim, 2014), which contains synapses between olfactory sensory nerve terminals and the apical dendrites of excitatory glutamatergic mitral cells (Fig. $1 A$, white cell bodies in the mitral cell layer, MCL). Layer-specific fMRI responses, however, cannot be concluded from this observation alone because the capillary density is also highest in GL (Borowsky and Collins, 1989; Korol and Brunjes, 1992; Lecoq et al., 2009; Poplawsky and Kim, 2014). Therefore, other olfactory bulb layers must be preferentially activated to differentiate neural-specific fMRI signal changes from the vascular distribution. To accomplish this, we stimulated the lateral olfactory tract (LOT) and the anterior com- missure (AC). Electrical stimulation of these fibers principally, but not exclusively, activates inhibitory GABAergic granule cells (Fig. 1A, black cell bodies in the granule cell layer, GCL). Specifically, LOT stimulation activates synapses at the distal dendrites of granule cells in the external plexiform layer (EPL, Fig. 1A, red circles), whereas AC stimulation mainly activates synapses at the proximal dendrites in GCL (Fig. 1A, blue circles) (Balu et al., 2007; Boyd et al., 2012; Markopoulos et al., 2012; Oswald and Urban, 2012).

In the present study, to clarify whether the neuronal and fMRI responses are spatially congruent, we examined fMRI signals evoked by layer-specific neuronal stimulation in the rat olfactory bulb. Odor, LOT, and AC stimulations independently evoked the greatest synaptic activity in GL (Fig. 1A, green circles), EPL (Fig. $1 A$, red circles) and GCL (Fig. 1A, blue circles), respectively. Because energy demand is highest for synaptic transmission (Attwell and Laughlin, 2001; Harris et al., 2012), we hypothesize that the largest hemodynamic responses will also be localized to these input layers.

\section{Materials and Methods}

Animal preparation and stimulating electrode placement. Fourteen male Sprague Dawley rats (280-510 g; $n=8$ for non-fMRI, $n=6$ for fMRI) were studied with approval from the University of Pittsburgh Institutional Animal Care and Use Committee in accordance with the standards for humane animal care and use as set by the Animal Welfare Act and the National Institutes of Health's Guide for the Care and Use of Laboratory Animals.

First, rats were induced with $5 \%$ and maintained with $2 \%$ isoflurane gas in a mixture of $30 \% \mathrm{O}_{2}$ and $70 \% \mathrm{~N}_{2}$ gases during surgery. The scalp between bregma and the olfactory bulb was removed and the skull was exposed to direct electrode placement, increase the coil sensitivity, and prevent image wrapping artifacts for the $7 \times 7 \mathrm{~mm}^{2}$ field of view (FOV). Small holes were made in the bone above the right olfactory bulb, left AC, right LOT, and right visual cortex. An $\mathrm{Ag} / \mathrm{AgCl}$ reference electrode was made by bleaching (catalog \#425044; Sigma-Aldrich) a silver wire (cata$\log$ \#50882; Stoelting), which was inserted between the dura and bone 
above the left visual cortex and fixed with acrylic dental cement. Tungsten stimulating electrodes (catalog \#577300 and \#575500; AM Systems; the electrode tips were sandpapered or cut to adjust the impedance to $<200 \mathrm{k} \Omega$ at $0.5 \mathrm{kHz})$ were located in the left $\mathrm{AC}(0.0 \mathrm{~mm}$ anteroposterior to bregma, $1.0 \mathrm{~mm}$ left of the midline, and $6.5 \mathrm{~mm}$ ventral to the brain surface; AP0.0/L1.0/D6.5) and right LOT (AP0.0/L4.5/D8.2) using stereotaxic instruments (SR-5R and SM-15; Narishige; Fig. $1 B, C$ ). In addition, a temporary recording electrode (catalog \#TM33A10; World Precision Instruments) was positioned in GCL of the right olfactory bulb $(\sim$ A8.0-9.0/L1.0/D1.0) to verify and optimize the placement of stimulating electrodes by evaluating the shapes and amplitudes of local field potentials (LFPs) evoked by AC and LOT stimulations (single $100 \mu \mathrm{s}$ pulse, $<-200 \mu \mathrm{A}$ amplitude, $1 \mathrm{~Hz}$ ). Isoflurane was temporarily reduced to $1.2-1.4 \%$ during LFP recordings. Electrical signals from the single electrode were amplified, digitized $(10 \mathrm{kHz})$, and filtered $(3-90 \mathrm{~Hz})$ with a neural recording system (MAP System; Plexon). After optimization, the stimulating electrodes were fixed in place with dental acrylic cement. The recording electrode was removed and the hole was sealed with $\sim 1 \%$ agarose followed by dental acrylic cement. After electrode implantation, the right femoral artery and vein were catheterized for physiological monitoring and administration of 5\% dextrose, anesthetic, and contrast agent, respectively. Carprofen $(4-5 \mathrm{mg} / \mathrm{kg}$, s.c.) as analgesia and atropine (0.05-0.07 mg/kg, i.m.) to prevent secretion and to open the nasal passages were administered at the beginning and end of surgery.

For hemodynamic measurements, isoflurane was discontinued and $\alpha$-chloralose dissolved in 2-hydroxypropyl- $\beta$-cyclodextrin (Storer et al., 1997) was administered ( $45 \mathrm{mg} / \mathrm{kg}$ i.v. induction, followed by a continuous $40 \mathrm{mg} / \mathrm{kg} / \mathrm{h}$ i.v. maintenance). Rats were free breathing and did not require intubation. Hemodynamic recording commenced $>1$ h (MP150; BioPac Systems) after the switch to $\alpha$-chloralose. The mean blood pressure was monitored through the arterial line and was maintained between $70-130 \mathrm{mmHg}$. In addition, the rat rectal temperature was maintained at $37 \pm 1^{\circ} \mathrm{C}$ using a warm water circulator and the breathing rate was recorded with a pneumatic pillow sensor. A $0.9 \%$ saline and 5\% dextrose supplemental fluid was administered intravenously at $1.0 \mathrm{ml} /$ $\mathrm{kg} / \mathrm{h}$.

Odor delivery and electrical stimulation of AC and LOT. A home-built olfactometer gated by data acquisitions was used for odor stimulation (Poplawsky and Kim, 2014). Briefly, a flow of $0.95 \mathrm{~L} / \mathrm{min}$ medical air and $0.05 \mathrm{~L} / \mathrm{min}_{2}$ gases was switched between a flask containing $100 \% \mathrm{~min}-$ eral oil or $5 \%$ amyl acetate in mineral oil and dedicated lines up to the rat snout allowed for quick transitions between the two conditions despite the long distance from the flasks to the magnet. For AC and LOT stimulations, a pulsed train of current (negative current of 150-200 $\mu \mathrm{A}, 10-80$ $\mathrm{Hz}, 100-200 \mu$ s pulse width) was delivered to the implanted monopolar stimulation electrode tips using an isolator (Isoflex; AMPI) equipped with an electrical pulse generator (Master 8; AMPI).

General experimental design. Two different experimental designs were used: (1) confirmation of layer-specific neuronal activations due to LOT and AC microstimulation by measuring evoked potentials across bulb layers and optimization of the stimulating frequencies of LOT and AC, and (2) hemodynamic responses of odor, LOT and AC stimulations measured by laser-Doppler flowmetry (LDF), BOLD fMRI, and cerebral blood volume-weighted (CBV) fMRI to determine the polarity of the hemodynamic responses induced by excitatory and inhibitory activities and the laminar specificity of hemodynamic responses.

After all experiments, a direct current $(-0.5 \mathrm{~mA}$ for $5 \mathrm{~s}$ or $-10 \mu \mathrm{A}$ for $30 \mathrm{~s}$ ) was applied to the AC and LOT stimulation electrodes to make electrolytic lesions at their tips. Rats were then killed with an intravenous injection of $0.5 \mathrm{ml}$ of saturated $\mathrm{KCl}$ under $>4 \%$ isoflurane and decapitated. The head was submerged in $10 \%$ formalin for at least $1 \mathrm{~d}$ and the brain was removed from the skull and immersed in either $4 \%$ paraformaldehyde or $10 \%$ formalin for $\sim 1$ week before cutting slices. Lesions in LOT and AC fibers were readily identified in $100-\mu \mathrm{m}$-thick slices without contrast staining. For histology of the bulb when identification of laminar structures was necessary, rats were deeply anesthetized and transcardially perfused with saline, followed by $4 \%$ paraformaldehyde. Slices ( $75 \mu \mathrm{m}$ thick) were then cut using a vibratome (Vibratome 1000 Plus; The Vibratome Company),
Nissl stained, and photographed to examine the recording sites and bulb layers.

Experimental design \#1: confirmation of layer-specific neuronal activity and optimization of stimulation frequency. To confirm the neuronal input layers due to LOT and AC electrical stimulations, current source density (CSD) maps were generated in one $\alpha$-chloralose anesthetized rat ( $308 \mathrm{~g}$ ). A recording electrode was inserted perpendicularly into the surface of the right olfactory bulb and placed $1.5 \mathrm{~mm}$ below the bulb surface. LFPs evoked by LOT and AC stimulation were then recorded (100 $\mu$ s pulse width, $-200 \mu \mathrm{A}$ amplitude, $1 \mathrm{~Hz}, 100$ repetitions) at this position. The same procedure was repeated at $100 \mu \mathrm{m}$ intervals dorsal to this position until the recording electrode reached the surface. After LFP recordings, electrolytic lesions were made (DC, $-10 \mu \mathrm{A}, 30 \mathrm{~s}$ ) at 2 recording depths ( $1.5 \mathrm{~mm}$ and $300 \mu \mathrm{m}$ below the bulb surface) to histologically identify the recording layers. The CSD was calculated from the average LFPs recorded at every $100 \mu \mathrm{m}$ depth using previously described methods (Vazquez et al., 2014).

To optimize LOT and AC stimulation frequencies, inhibition of spontaneous mitral cell output by evoked granule cell activity was recorded in MCL from five rats (two sites per rat). Stimulations consisted of a $10 \mathrm{~s}$ off, $10 \mathrm{~s}$ on, and $10 \mathrm{~s}$ off period. Stimulation frequencies (100 $\mu$ s pulse width and $-150 \mu \mathrm{A}$ amplitude at 10, 20, 40 and $80 \mathrm{~Hz}$ ) were pseudorandomized and repeated 10 times for each frequency. First, optical imaging of intrinsic signal was performed to identify the locations of active glomeruli "hot spots" evoked by $5 \%$ amyl acetate (data not shown). Two tungsten electrodes were then inserted into the hot spots to record multi-unit activities (MUAs) from MCL. The raw electrophysiological signal was recorded at $10 \mathrm{kHz}$ and band-pass filtered $(400 \mathrm{~Hz}-9 \mathrm{kHz})$. A single spike event (limit of 1 per $1.8 \mathrm{~ms}$ block) was counted when it exceeded a threshold of 3 SDs from the average of the $10 \mathrm{~s}$ prestimulus background signal. The peristimulus time histogram (PSTH) was constructed from the spike counts using a $100 \mathrm{~ms}$ bin. An $8 \mathrm{~ms}$ period after each stimulation onset was omitted from spike detection to exclude the artifact elicited by the electrical stimulation and the evoked antidromic and GCL population spikes (Fig. 2C, left). Because this removal will also result in fewer counted spikes during the stimulation period, these $8 \mathrm{~ms}$ periods were also removed from the $10 \mathrm{~s}$ prestimulation and poststimulation periods every $100 \mathrm{~ms}$ at $10 \mathrm{~Hz}, 50 \mathrm{~ms}$ at $20 \mathrm{~Hz}, 25 \mathrm{~ms}$ at $40 \mathrm{~Hz}$, and $12.5 \mathrm{~ms}$ at $80 \mathrm{~Hz}$ to control for this effect before generating the PSTH. The percent change of the firing rate was then calculated from the mean of the prestimulus baseline.

For LDF measurements, two LDF probes ( $450 \mu \mathrm{m}$ in diameter, time constant $=0.03 \mathrm{~s}$, Periflux 5000; Perimed) were placed on the dorsal surface of the bulb in the vicinity of the hot spots to record CBF. The LDF probes were generally $>1 \mathrm{~mm}$ apart and located in anterior and posterior parts of the olfactory bulb. A pulsed train of current $(-150 \mu \mathrm{A}$ and 100 $\mu$ s pulse width at $40 \mathrm{~Hz}$ for $64 \mathrm{~s}$ ) was delivered to the implanted monopolar stimulation electrode tips in AC and LOT. Odor (5\% amyl acetate), AC, and LOT stimulations were interleaved and repeated 5-10 times. A single run for all the stimulation paradigms consisted of $120 \mathrm{~s}$ off, $64 \mathrm{~s}$ on, and $120 \mathrm{~s}$ off periods.

Experimental design \#2: BOLD and CBV fMRI. All MRI experiments were performed on a $9.4 \mathrm{~T} / 31 \mathrm{~cm}$ MR system interfaced by a DirectDrive console (Agilent Technologies) and an actively shielded gradient coil with $40 \mathrm{G} / \mathrm{cm}$ peak gradient strength and $120 \mu$ s rise time (Magnex). The head of the rat was fixed in a nonmagnetic head restraint with a bite bar and ear plugs. A custom-built, $1 \mathrm{~cm}$ inner diameter surface coil was positioned dorsal to the olfactory bulb for radiofrequency excitation and reception. Both removal of the dorsal scalp and the diminished coil sensitivity in the ventral bulb minimized folding artifacts in the dorsoventral phase-encoding direction.

Anatomical MRI. Olfactory bulb layers were defined on high-resolution anatomical images and verified with classical histological staining methods in one rat $(303 \mathrm{~g})$. Anatomical images were acquired using a fast spin-echo sequence and had a $128 \times 128$ matrix size, $7 \times 7 \mathrm{~mm}^{2}$ FOV $\left(55 \times 55 \mu \mathrm{m}^{2}\right.$ in-plane resolution), $3.0 \mathrm{~s}$ repetition time (TR), train of 4 echoes, $40.7 \mathrm{~ms}$ effective echo time (TE), 90.5 -mm-thick slices with no gap, and 24 averages. In addition, anatomical images with a thinner slice thickness were acquired 
to assess out-of-plane layer identifications: $96 \times$ 96 matrix size, a $7.7 \times 7.7 \mathrm{~mm}^{2}$ FOV $(80 \times 80$ $\mu \mathrm{m}^{2}$ in-plane resolution), $23 \quad 0.2$-mm-thick slices, and 108 averages. Next, the rat was perfused and $75-\mu \mathrm{m}$-thick slices of the bulb were Nissl stained. The anatomical MRI and Nissl stained images were qualitatively compared to verify layer definitions with the endogenous MRI contrasts.

fMRI data acquisition. We measured two types of fMRI contrasts: endogenous BOLD, followed by contrast-enhanced CBV fMRI [single intravenous bolus of Feraheme (ferumoxytol; AMAG Pharmaceuticals), $15 \mathrm{mg}$ of $\mathrm{Fe} / \mathrm{kg}$ ] using a recently developed compressedsensing, gradient-recalled echo sequence to increase the temporal resolution by four times (Zong et al., 2014). In six rats (280-420 g), fMRI images during odor, LOT, and AC stimulations were acquired with the same FOV as the anatomical images $\left(7 \times 7 \mathrm{~mm}^{2}\right), 64 \times 64$ matrix size $\left(110 \times 110 \mu \mathrm{m}^{2}\right.$ in-plane resolution), $125 \mathrm{~ms}$ TR, $18 \mathrm{~ms}$ (BOLD) or $8 \mathrm{~ms}$ (CBV) TE, compressed sensing reduction factor of 4 ( 16 of 64 phase encode lines were measured), 5 (BOLD) or 9 (CBV) 0.5-mm-thick slices, 32 dummy scans, and $2 \mathrm{~s}$ temporal resolution. The phase encode undersampling pattern was chosen due to its increased contrastto-noise ratio compared with other patterns and consisted of a constant sampling of the six center $k$-space lines plus a random sampling of 10 of the remaining 58 lines for each time point (Rand+C6). A $10 \mathrm{kHz}$ sampling bandwidth was chosen for its increased baseline signal-tonoise ratio (SNR). The optimal flip angle was determined by acquiring the baseline fMRI images at increasing flip angles and selecting the one that had the maximal signal within the olfactory bulb. Images were reconstructed using a $k-t$ FOCUSS algorithm with a KarhunenLoeve sparsifying transform (FOCUSS iteration number $=2$, iteration number of conjugate gradient $=60$, weighting matrix update power $\gamma=0.5$, and regularization factor $\lambda=0.01$ ). A single fMRI run consisted of 60 baseline $(120 \mathrm{~s}), 32$ stimulus-evoked (64 s), and 60 recovery (120 s) images. A single stimulation cycle consisted of $15 \%$ amyl acetate odor run, followed by 1 left AC run and 1 right LOT run $(-200 \mu \mathrm{A}$ and $200 \mu$ s pulse width at $40 \mathrm{~Hz})$. Each stimulation cycle was repeated six times for a total of 18 fMRI runs. Runs within the same cycle had the same Rand + C6 sampling patterns, whereas different random patterns were used between cycles. The time between stimulation offset to onset of the subsequent run was $\sim 4$ min and the subsequent cycle (i.e., stimulation of the same type) was $\sim 19 \mathrm{~min}$.

General fMRI data analysis. Data were preprocessed and individual functional maps were calculated using SPM8 (Wellcome Trust Centre for Neuroimaging, London). In addition, a quantitative layer-specific analysis was performed on a region-of-interest (ROI) basis and a group analysis between rats was performed using SPM8.

Single animal functional maps. Reconstructed images were spatially realigned, linearly detrended, and the normalized difference of the fMRI series was calculated $\left(\left[S_{\mathrm{t}}-S_{0}\right] / S_{0}\right.$, where $S_{0}$ is the mean of repetition numbers 1-60 and 148-152) using home-written Matlab code (The MathWorks). Preliminary analysis showed no motion to stimulations and negligible motion within the timespan of a single trial $(\sim 5 \mathrm{~min})$, whereas, in some rats, slow spatial drifting $(\sim 1-2$ total in-plane voxels) was observed over the course of the entire fMRI experiment ( $\sim 140 \mathrm{~min})$.

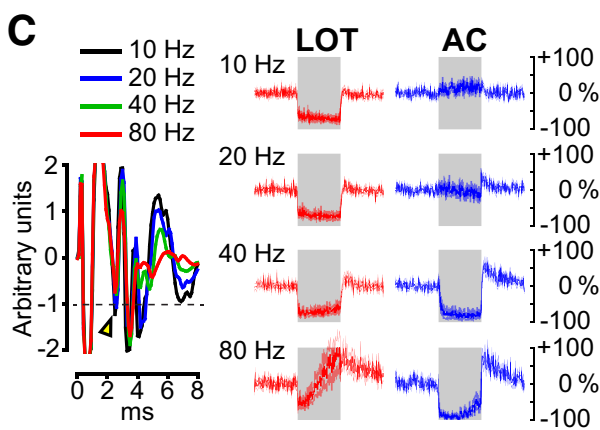

D

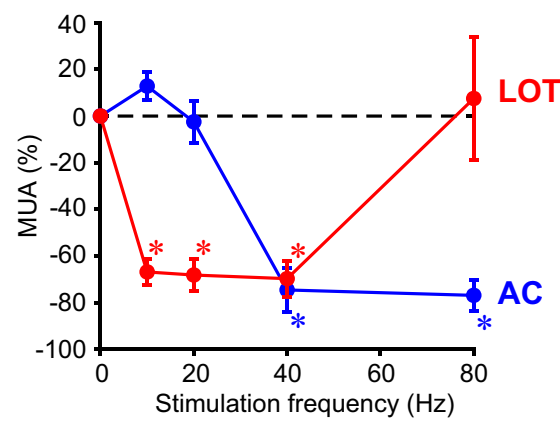

Figure 2. Confirmation and optimization of layer-specific neuronal activity. $\boldsymbol{A}$, Right LOT and left AC stimulations (indicated by a downward arrow) evoked granule cell activity to produce signature unilateral and bilateral LFPs, respectively (mean \pm SD of 10 runs, dashed vertical line separates the left and right bulb recordings). Note that the antidromic potential ( $\sim 2$ ms latency after the 列 , 10 ms. C, Mean time courses of stimulation-frequency-dependent suppression rates of spontaneous MUAs restimulus baseline) $\mathrm{MCL}$ was determined by the reversal of $\mathrm{LOT}$ stimulation-evoked LFP (B) Gray bars indicate a 10 stimulation with a $100 \mu$ s pulse width and a $-150 \mu \mathrm{A}$ amplitude at $10,20,40$, and $80 \mathrm{~Hz}$. Inset, Confirmation of antidromic spikes (yellow arrowhead). Average for 10 runs and individual pulses delivered to LOT (i.e., 100, 200, 400, and 800 pulses for 10 Suppression of the mean firing rate is plotted as a function of rats). Mean firing rate of the $10 \mathrm{~s}$ prestimulation baseline was compared with that of a $10 \mathrm{~s}$ stimulation period (negative and for $\mathrm{AC}$ stimulation at $10 \mathrm{~Hz}(17.2 \pm 7.9 \%, p=0.101)$, possibly due to mitral cell disinhibition (Boyd et al., 2012), and returned to baseline during LOT stimulation at $80 \mathrm{~Hz}$, possibly due to slow kinetics of targeted synapses, short-term plasticity, or presynaptic glutamate depletion. ${ }^{*} p<0.01$.

Because motion over any one run was negligible, realignment was performed on a run-by-run basis by coregistering the average fMRI image of each run to the average fMRI image of the first run using a rigid-body, three degree-of-freedom transformation (3-DOF: translations in $x$ - and $y$-axes and rotation about the $z$-axis). The estimated motion parameters for each run were then applied to all of the corresponding fMRI time points. Individual trials were grouped according to their stimulation type and the corresponding time series were concatenated for subsequent analysis in SPM8.

Percent signal change and $t$-value maps were calculated using SPM8, which uses a general linear model to estimate the effects $(\beta)$ of a predicted design matrix $(X)$ from the experimental measurements $(Y): Y=\beta X+E$, where $E$ is an estimate of error. The fMRI time points $\times 2$ design matrix consisted of a constant, baseline variable $\left(X_{\text {baseline }}\right)$ and a predicted hemodynamic response function $\left(X_{\mathrm{HRF}}\right)$ that was calculated by convolving the concatenated block-stimulation paradigm with the default SPM8 BOLD HRF and a previously reported CBV impulse response function (Silva et al., 2007). The effect estimates for each respective variable ( $\beta$-maps) were then calculated from the preprocessed and concatenated fMRI time series $(Y)$. We also chose an autoregressive $[\mathrm{AR}(1)]$ model for the unpredicted serial correla- 
tions, and a classical estimation of model parameters using restricted maximum likelihood methods in SPM8. The resulting $\beta$-maps were then used to calculate the percent signal change $\left(100 \% * \beta_{\mathrm{HRF}} / \beta_{\text {baseline }}\right)$ and $t$-value functional maps. These functional maps were coregistered to the anatomical images and linearly interpolated to a $128 \times 128$ matrix size to match the anatomical space.

Group analysis. To perform voxelwise statistical testing across rats, individual functional maps were normalized to a similar anatomical space using SPM8. These transformations were estimated using the anatomical image volumes from each rat with 1 representative rat serving as the template (default parameters were chosen except for reference image smoothing $=1 \mathrm{~mm}$, source image smoothing $=1 \mathrm{~mm}$ and affine regularization $=$ average sized template). The resulting six coregistered anatomical volumes were then averaged and subsequently used as the mean anatomical underlay for the group functional maps. Next, the estimated transformations were applied to the corresponding functional maps (previously registered to the anatomical images) and analyzed in SPM8 using a within-subject one-way ANOVA second-level analysis. Group $t$-maps were calculated for each stimulus in SPM8.

Line profiles were obtained to compare laminar patterns between stimuli without a statistical threshold. Straight lines were drawn from the lateral surface of the left bulb through the right at a dorsoventral depth (approximately halfway between the dorsal and ventral extremes) where the layers ran orthogonal to the line. Points were then averaged in the dorsoventral direction corresponding to a line width of $330 \mu \mathrm{m}$ before plotting.

ROI analysis. ROIs were manually drawn on the high-resolution anatomical images of individual rats and corresponded to the seven layers previously verified with histological staining methods. ROIs were drawn on the dorsal $\sim 2 / 3$ of all 5 BOLD fMRI slices and the corresponding 5 CBV fMRI slices because the ventral $\sim 1 / 3$ of slices had a diminished coil sensitivity that caused a less reliable layer identification (characterized by an approximate $40 \%$ decrease in SNR relative to the dorsal bulb). The surface ROI included the bulb surface, midline, and any pial vessels identified as signal hypo-intensities, GL and MCL were defined by the outer and inner bands of hypo-intense signal, respectively, and core by the innermost hyperintense band, whereas olfactory nerve layer (ONL), EPL, and GCL were identified by their spatial relationship to these bands (Poplawsky and Kim, 2014). No voxel in the ROI mask was assigned to more than one ROI and some remaining partial volume effects are expected at the ROI boundaries due to the limited spatial resolution.

Two ROI analyses were performed: (1) the mean percent signal changes, $t$-values, and time courses of unthresholded individual rat data were calculated for each of the seven ROIs for each stimulus in each bulb and (2) each of these ROI values, except for the time course data, was normalized by the mean absolute value of all seven ROIs in each bulb (a measure of entire bulb activity whether positive or negative) to adjust for interbulb and interstimulus variations. Finally, the mean value for each ROI across the 12 bulbs was calculated for odor and AC stimulations, whereas the six right and six left bulbs were separately considered for LOT stimulation because almost no activation was expected in the contralateral bulb. Differences between stimulations were then compared with a one-way ANOVA and post hoc Student's $t$ tests.

\section{Results}

Confirmation of stimulating electrode positions by electrophysiology and optimization of stimulation frequency

Before fMRI experiments, monopolar stimulating electrodes were stereotaxically guided to the right LOT and left AC (Fig. 1B) and implanted at optimal positions based on the LFPs evoked in the bulb GCL (Fig. 2A). LOT stimulation evoked a brief negative potential in the right bulb, indicating antidromic activation of excitatory mitral cells (the solid arrowhead with the number 1 label on the red trace), followed by large positive (labeled 2) and negative potentials (labeled 3 ), representing excitation of inhibitory granule cells (Rall and Shepherd, 1968; Nakashima et al., 1978; Uva et al., 2006). Conversely, AC stimulation evoked a negative potential, with two peaks (Fig. $2 A$, blue traces) indicat- ing monosynaptic and multisynaptic excitation of the granule cells (Mori and Takagi, 1978; Nakashima et al., 1978; Nickell and Shipley, 1993). LOT stimulation evoked LFPs in the bulb ipsilateral to the stimulation only, whereas AC stimulation evoked LFPs bilaterally (Fig. 2A). Therefore, if activation of inhibitory neurons evokes a hemodynamic response, LOT and AC stimulations are expected to evoke unilateral and bilateral fMRI responses, respectively. In addition, CSD maps were calculated from the evoked LFPs at different bulb depths and confirmed the location of current sinks (blue in the CSD maps) due primarily to synaptic inputs at EPL for LOT and GCL for AC stimulations (Fig. 2B; see also Laaris et al., 2007). To activate inhibitory neurons maximally, we first determined the optimal stimulation frequency that minimized the spontaneous MUAs at MCL for 10-s-long LOT and AC stimulations with $-150 \mu \mathrm{A}$ amplitude and $100 \mu$ s pulse width (Fig. 2C,D). MCL was first identified by LFPs evoked by LOT stimulation in which the polarity of the postsynaptic LFP components reversed at MCL (Rall and Shepherd, 1968; Nickell and Shipley, 1988; Fig. 2B). For LOT stimulation, the amplitudes of the first evoked spikes, the onset of which preceded the onset of the initial negative deflection of the LFP at MCL (onset, $1.7 \pm 0.1$ ms; peak, $2.5 \pm 0.1 \mathrm{~ms}, n=10$ ), were also checked for the fidelity of antidromic mitral cell activation. If the spikes are antidromically evoked, their peak amplitudes should be constant with increasing stimulation frequencies. However, their amplitudes more or less decreased as the stimulation frequency increased (Fig. 2C, left, yellow arrowhead), suggesting that the evoked population spikes are not purely antidromic. This also suggests that the initial negative deflection of the LFP evoked by LOT stimulation (Fig. 2A) may not be entirely composed of antidromic and presynaptic events, contrary to what Rall and Shepherd (1968) suggested. MUA suppression rates for different stimulation frequencies were different between LOT and AC stimulations (Fig. $2 C, D)$. Spontaneous MUAs at MCL were significantly $(p<0.01)$ suppressed during 10 -s-long stimulations at $10 \mathrm{~Hz}(66.9 \pm 5.6 \%$, $n=10$ sites from 5 rats, mean \pm SEM unless otherwise indicated), $20 \mathrm{~Hz}(68.2 \pm 6.8 \%)$, and $40 \mathrm{~Hz}(69.7 \pm 7.7 \%)$ for LOT stimulation, whereas MUAs were significantly $(p<0.01)$ suppressed only at $40 \mathrm{~Hz}(74.6 \pm 9.4 \%)$ and $80 \mathrm{~Hz}(76.9 \pm 6.7 \%)$ for AC stimulation. We chose $40 \mathrm{~Hz}$ for both LOT and AC stimulations in the following experiments.

To test whether LOT and AC stimulations elicited CBF responses, as with odor stimulation, CBF was recorded from the bulb surface using two LDF probes concurrently with MUA recordings at MCL in the right bulb (Fig. 3). Monitoring the mitral cell spiking activity ensures stimulation efficacy of each pathway because the mitral cell is the common target of the three neural circuits. Particularly, it is useful as an indicator of GABAergic neuronal activity during LOT and AC stimulations.

First, we confirmed that the average odor-evoked (5\% amyl acetate in mineral oil) CBF (18.8 $\pm 2.8 \%$, average for $0-64 \mathrm{~s}, n=$ 10 recording sites from 5 rats) and MUA responses (31.9 \pm $14.8 \%$, average for $0-64 \mathrm{~s}, n=10$ ) were increased (Fig. 3, left panels). Although the CBF responses consistently increased for all 10 recording sites, the spiking activity of mitral cells was more variable: compared with the $120 \mathrm{~s}$ prestimulus baseline using two-sample $t$ tests, the mean MUA for the entire 0-64 s stimulation period showed significant increases at 8 sites $(41.5 \pm 15.1 \%$, $p<0.01)$, decreases at 1 site $(-15.1 \%, p<0.01)$, and no change at 1 site $(p=0.319)$ from baseline. This discrepancy is likely due to sample volume mismatches between MUA and CBF recording sites. For both AC and LOT stimulations, we sometimes observed mean arterial blood pressure (MABP) changes (Fig. 3, top), but 

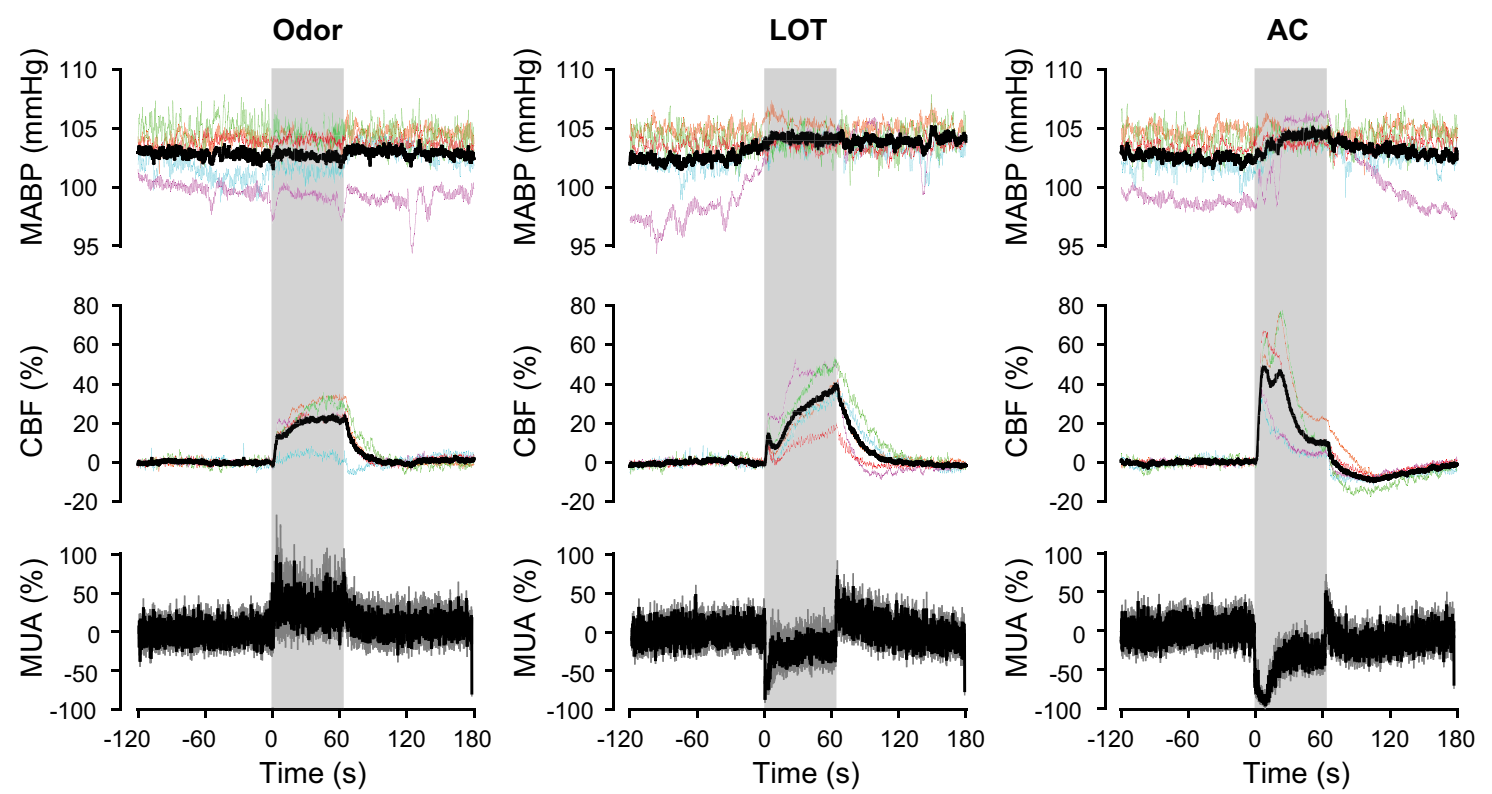

Figure 3. CBF increases with decreasing spontaneous MUA in MCL during the 64-s-long LOT and AC stimulations. Time courses of the mean arterial blood pressure (MABP, top), CBF (middle), and relative MUA changes (bottom) are shown for odor stimulation (left) and electrical stimulations of LOT (middle) and AC (right; - $150 \mu \mathrm{A}$ amplitude, $100 \mu$ s pulse width, $40 \mathrm{~Hz}$ ). CBF responses were obtained from two different sites, located $>1 \mathrm{~mm}$ apart. For clarity, the averaged time courses of these two sites were shown because they had similar shapes $(r$, Pearson's linear correlation between the two LDF signals, $n=5$ rats: $0.96 \pm 0.02, p<0.01$ for odor, $0.98 \pm 0.00, p<0.01$ for L $0 T, 0.96 \pm 0.03, p<0.01$ for AC stimulations). For (BF and MABP traces, data from the same rat are coded with the same color, whereas the black traces are the averages of the 5 rats. For MUA time courses, all data points were normalized to the mean firing rate of the $120 \mathrm{~s}$ prestimulation baseline at each recording site and their averages (mean $\pm \mathrm{SEM}, n=10$ sites from 5 rats) are shown (negative and positive changes represent percentage decreases and increases from baseline, respectively). Vertical gray bars, $64 \mathrm{~s}$ stimulation period.

these were short and negligibly small $(0.2 \pm 0.2 \%$ for AC and $1.5 \pm 1.0 \%$ for LOT, $n=5$ rats, during the $0-10 \mathrm{~s}$ period relative to baseline). Therefore, the MABP changes did not seem to affect the evoked CBF responses. Electrical stimulations of each tract $(-150 \mu \mathrm{A}$ amplitude, $100 \mu$ s pulse width, $40 \mathrm{~Hz})$ increased CBF and decreased the spontaneous MUAs of mitral cells, thus confirming increased evoked GABAergic neuronal activity. For LOT stimulation (Fig. 3, middle panels), the average CBF increased and reached the first peak $(14.5 \pm 2.5 \%$ at $3.8 \mathrm{~s}, n=10$ recording sites) during the initial strong MUA suppression (e.g., $-64.6 \pm$ $6.2 \%$ for $0-3 \mathrm{~s}, p<0.01 ;-57.8 \pm 8.2 \%$ for $0-5 \mathrm{~s}, p<0.01$; $-42.9 \pm 14.8 \%$ for $0-10 \mathrm{~s}, p=0.03$ ) and returned quickly toward baseline before it started to gradually increase until the end of the stimulation ( $38.2 \pm 4.5 \%$ at $64 \mathrm{~s}, n=10)$. During this CBF gradual development, the MUA suppression became weaker $(-22.0 \pm 13.2 \%$ for $10-64 \mathrm{~s}, p=0.14$; significant decreases at 6 sites $(-51.4 \pm 8.0 \%, p<0.01)$, increases at 3 sites $(28.2 \pm 9.2 \%$, $p<0.01)$, and no change at 1 site $(p=0.364))$. Therefore, the $\mathrm{CBF}$ response did not seem to completely follow the MUA suppression caused by the excitation of the GABAergic neurons. Conversely, for AC stimulation (Fig. 3, right panels), the CBF response was more similar to the MUA suppression. The average $\mathrm{CBF}$ increased and peaked $(48.6 \pm 5.7 \%$ at $7.3 \mathrm{~s}, n=10$ during the initial strong MUA suppression (e.g., $-78.0 \pm 5.3 \%$ for $0-10$ $\mathrm{s}, p<0.01 ;-72.6 \pm 6.3 \%$ for $0-20 \mathrm{~s}, p<0.01)$ and started to decrease when the MUA suppression became weaker $(-31.4 \pm$ $5.9 \%$ for $20-64 \mathrm{~s}, p<0.01)$. It should be noted that spontaneous MUAs were transiently increased after the long suppression during AC and LOT stimulations likely due to rebound discharges of mitral cells (Balu and Strowbridge, 2007).

Odor, AC, and LOT stimulations increase the fMRI responses The LDF results indicated that both LOT and AC stimulations increased the CBF responses in the bulb. However, our LDF probes measured CBF changes mainly from the bulb surface and not from the deeper layers where the evoked neuronal activity was located. Therefore, we measured fMRI changes in the entire bulb during LOT and AC stimulations, as well as odor stimulation, to include hemodynamic changes in the same layers as the evoked neuronal activity. We delivered odor (5\% amyl acetate for $64 \mathrm{~s})$ to both nostrils with a home-built olfactometer and electrically stimulated LOT and AC $(-200 \mu \mathrm{A}$ amplitude, $200 \mu$ s pulse width, $40 \mathrm{~Hz}, 64 \mathrm{~s}$ duration) using the implanted electrodes during fMRI. We measured the BOLD and CBV fMRI changes to these 3 separate stimulations at $9.4 \mathrm{~T}$ and at high spatial $(110 \times$ $110 \times 500 \mathrm{\mu m}^{3}$ ) and temporal resolutions ( $2 \mathrm{~s}$, compressed sensing gradient-recalled echo fMRI; Zong et al., 2014) in 6 rats. BOLD data during LOT stimulation were removed in one rat due to inconsistent stimulus delivery. We calculated statistical and percent change maps using a general linear model analysis in SPM8. Because a decrease in the raw CBV fMRI signal indicates an increase in blood volume, the signs of the CBV fMRI signal changes were inverted to facilitate comparisons with BOLD. Therefore, positive CBV fMRI responses represent blood volume increases and negative changes represent decreases. We consistently observed that both BOLD and CBV responses increased for odor, LOT, and AC stimulations for all rats (see Fig. 4 for a single representative animal). The fMRI signal changes of all voxels in each bulb were averaged and compared with a $0 \%$ signal change across bulbs using a 1 -sample $t$ test. The average BOLD signal changes across bulbs were as follows: odor $=+1.0 \pm$ $0.08 \%(p<0.001, n=12$ left and right bulbs $), \mathrm{LOT}=+1.6 \pm$ $0.3 \%(p<0.001, n=5$ right bulbs $)$, and $\mathrm{AC}=+0.3 \pm 0.1 \%$ $(p=0.013, n=12)$; for CBV: odor $=+2.4 \pm 0.2 \%(p<$ $0.001, n=12), \mathrm{LOT}=+1.5 \pm 0.4 \%(p=0.004, n=6$ right bulbs $)$, and $\mathrm{AC}=+1.3 \pm 0.1 \%(p<0.001, n=12)$. These indicate that BOLD and CBV fMRI responses increased for all stimulations. On the contrary, the fMRI responses in the left 
A

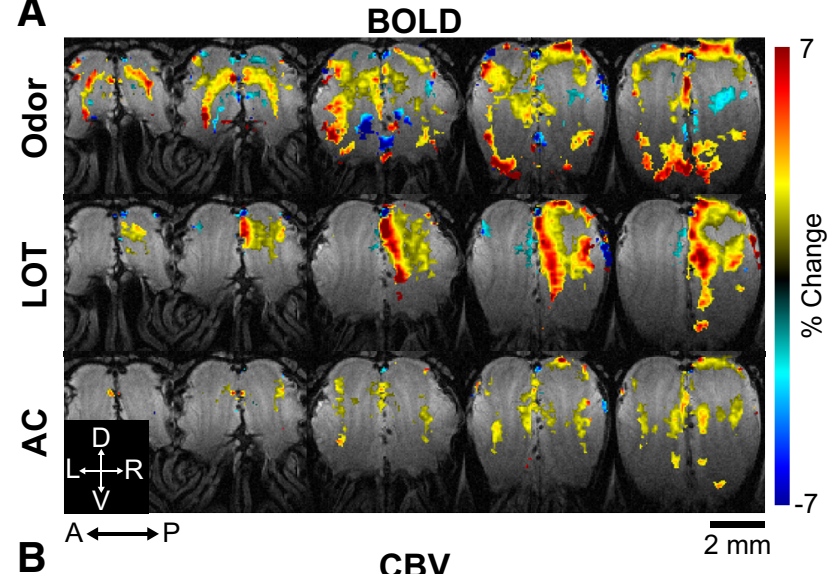

B

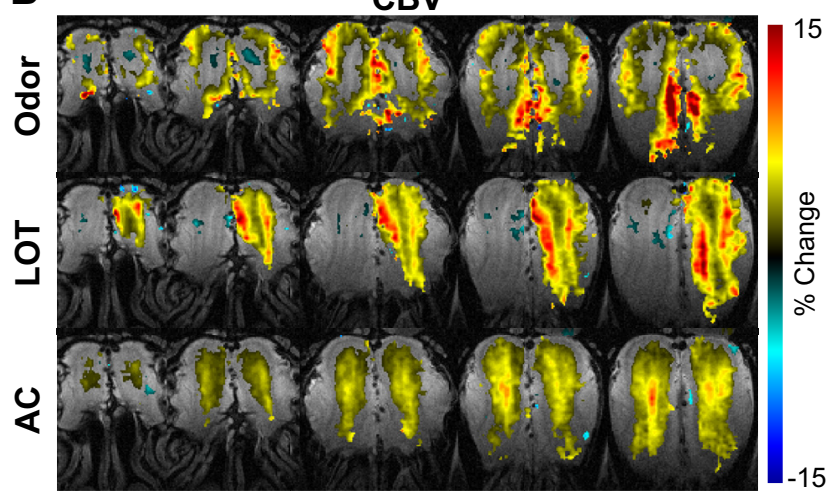

Figure 4. Odor, $L O T$, and $A C$ stimulations all increase the $B O L D$ and $C B V$ fMRI responses. $A, B$, BOLD $(\boldsymbol{A})$ and $\mathrm{CBV}(\boldsymbol{B}) \mathrm{fMRI}$ responses in five coronal slices of one representative animal increase for all three stimulations ( $p<0.01$ (voxelwise) and minimum number of clustered active voxels equal to 21 for a familywise error correction of $p<0.01$ ). To compare with BOLD fMRI, the polarity of raw CBV fMRI data was inverted; therefore, positive CBV fMRI signal changes represent blood volume increases. Refer to Figure 6 for group fMRI maps that represent the entire sample population. A, Anterior; $P$, posterior; $D$, dorsal; $V$, ventral; $L$, left; $R$, right.

bulb after LOT stimulation showed small negative or no changes (BOLD: $-0.11 \pm 0.05 \%, p=0.031, n=5$; CBV: $-0.01 \pm 0.12 \%, p=0.920, n=6)$, which is consistent to a lack of major evoked neuronal responses here (Fig. 2A).

BOLD fMRI responses are poorly localized to the layer of neuronal activity

We then investigated whether the increased hemodynamic responses occur at the same location as the layer-specific evoked synaptic activity. To assess the layer specificity of the fMRI response, the bulb layers were first identified on high-resolution anatomical MRI images $\left(55 \times 55 \times 500 \mu \mathrm{m}^{3}\right)$. Alternating dark and light bands identified on anatomical MRI images were consistent with the laminar definitions using traditional Nisslstained histological images in the same rat (Fig. 5A, $n=1$ rat). Increasing the image slice thickness will increase the baseline SNR, but will also increase the partial volume effects that could cause blurring of the layers. We chose a $500 \mu \mathrm{m}$ slice thickness to balance these concerns, since a 500- $\mu \mathrm{m}$-thick slice had similar laminar assignments as the corresponding 200- $\mu \mathrm{m}$-thick slices in the same rat (Fig. $5 B-D$ ). In addition, the mean baseline SNR (average signal intensity/SD) increased from superficial to deep bulb layers for BOLD (e.g., $11.4 \pm 0.6$ for GL, $15.5 \pm 0.9$ for EPL, $18.8 \pm 1.3$ for GCL, $n=12$ bulbs) and CBV (e.g., $12.1 \pm 0.6$ for GL, $19.3 \pm 1.0$ for EPL, $25.3 \pm 1.3$ for GCL, $n=12$ bulbs) fMRI

\section{A Anatomical MRI}
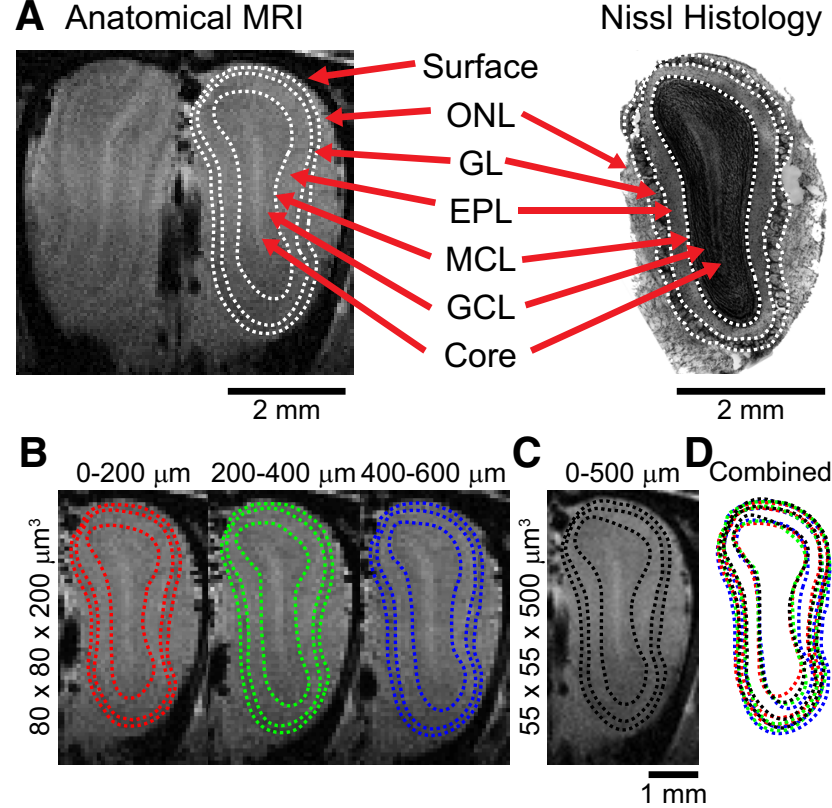

Figure 5. Histological confirmation of anatomical MRI layer definitions and determination of fMRI slice thickness. $\boldsymbol{A}$, Layers defined on the anatomical MRI slices (500 $\mu \mathrm{m}$ thick) were histologically verified (Nissl stain, $75 \mu \mathrm{m}$ thick) in the same rat. Dashed lines represent the inner and outer boundaries of GL and the outer boundary of MCL. In addition, anatomical MRI images were acquired at $200 \mu \mathrm{m}(\boldsymbol{B})$ and $500 \mu \mathrm{m}(\boldsymbol{C})$ slice thicknesses to assess the out-of-plane partial volume effects on laminar definitions. The relative anteroposterior positions are indicated above each slice. When combined $(\boldsymbol{D})$, the layers are highly overlapped, especially in the dorsal two-thirds of the bulb. The layers in slice position $400-600 \mu \mathrm{m}$ ( $\boldsymbol{B}$, blue outlines) begin to diverge from those in slice position $0-500 \mu \mathrm{m}$ ( $\boldsymbol{C}$, black), suggesting an upper limit of 500 $\mu \mathrm{m}$ slice thickness for subsequent laminar fMRI studies.

contrasts. Therefore, the SNR profiles across layers are opposite to the baseline total and microvessel blood volumes, which decrease with bulb depth (Poplawsky and Kim, 2014).

The hypothesized patterns of layer-specific synaptic inputs for each stimulus (indicated in Fig. $6 A$ by colored bands) were compared with the actual laminar fMRI activation patterns for each fMRI contrast using the same trichromatic color scheme. In the group BOLD fMRI statistical maps (Fig. 6B), we observed widespread activity in the superficial and middle layers for both odor and LOT stimulations, with some overlap when combined (Fig. $6 B$, yellow). Conversely, AC stimulation produced a sparse activation pattern that spanned most of the bulb layers. For all three stimulations, layer-specific activation patterns are not competently separable. The spatial specificity of the BOLD signal in the group maps was further examined without the influence of statistical thresholds by way of a $330-\mu$ m-width line profile in the posterior-most slice. At this example location, a line can be drawn across the left and right bulbs where the line cuts approximately orthogonal to the bulb layers and the layers are thicker compared with anterior slices. At this specific bulb location, functional peaks for odor and LOT stimulations were broad and spatially overlapped, whereas the weak AC signal changes make it difficult to conclude whether preferential laminar activation is present (Fig. 6C). To better analyze the spatial distributions of the hemodynamic responses independent of the line position chosen, ROI analyses were performed on the entire bulbs for odor, LOT, and AC stimulations (Fig. 7A,B). The magnitudes of all voxels within each ROI of the seven bulb layers, defined on anatomical images, were averaged and normalized by the mean absolute signal 
A
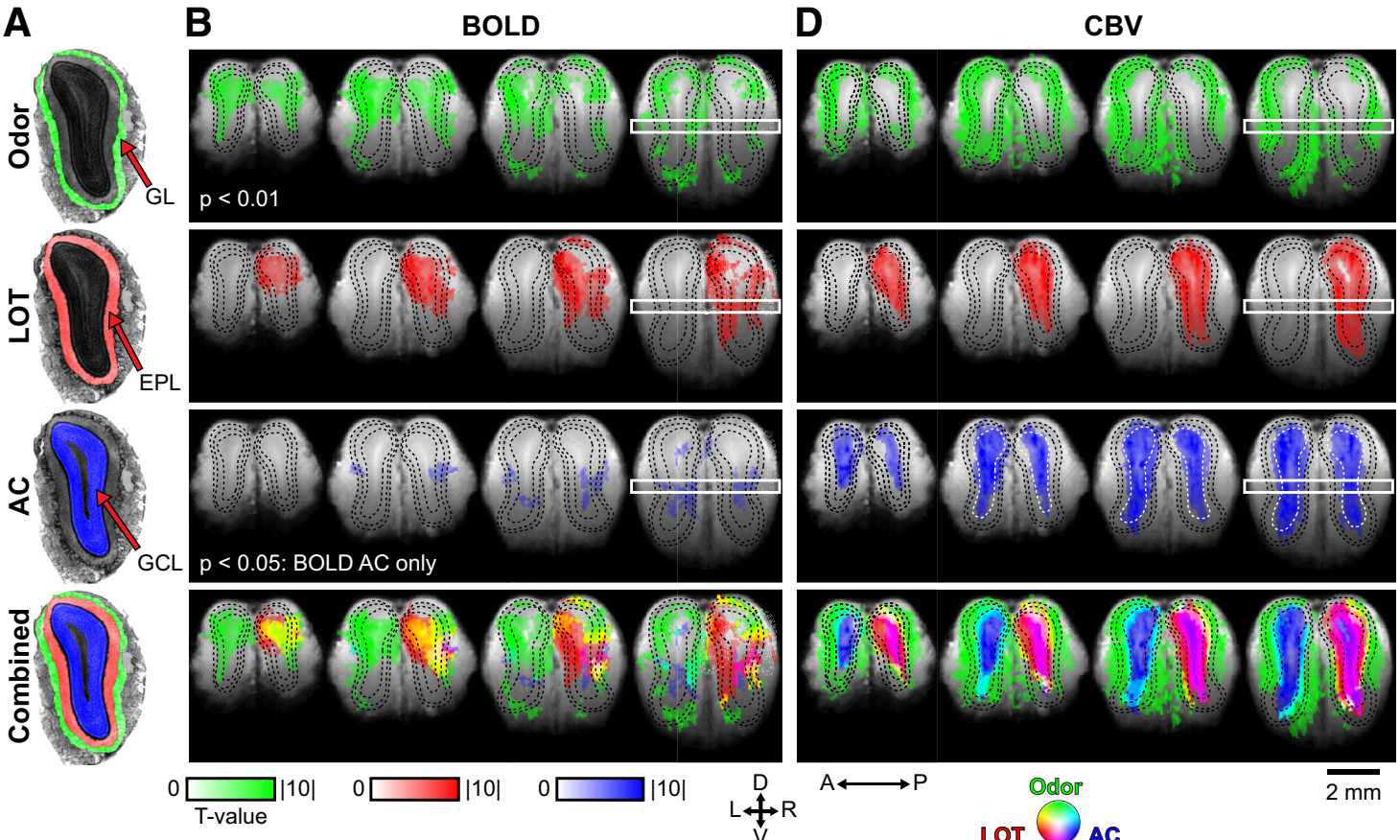

C
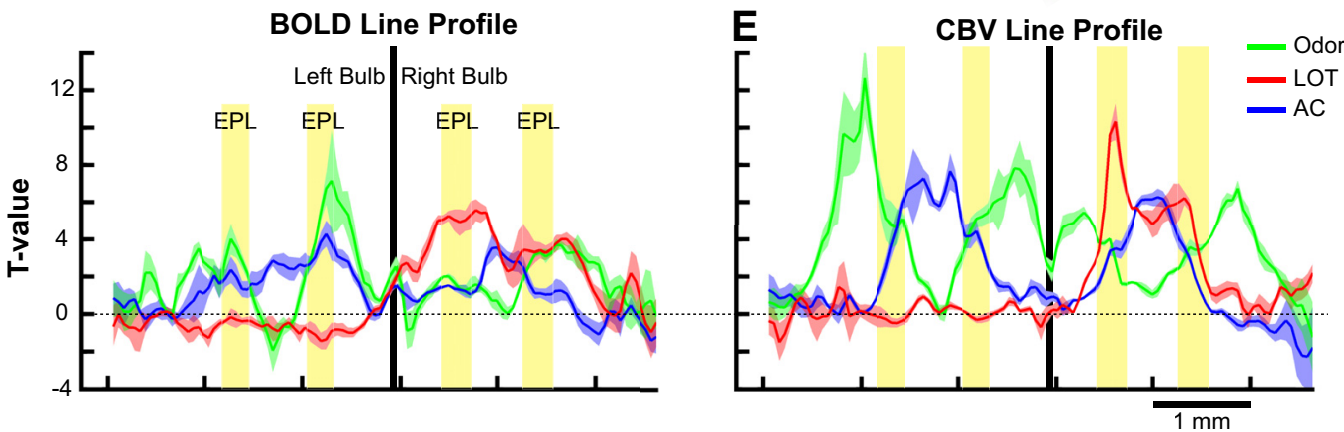

Figure 6. CBV fMRI responses are specific to the site of synaptic activity at laminar resolutions, whereas BOLD changes are poorly localized. $A$, Primary locations of evoked synaptic activity are hypothesized to be the location of the maximal fMRI responses and are color coded in the Nissl-stained image: odor stimulation in GL (green), LOT in EPL (red), and AC in GCL (blue). $\boldsymbol{B}, \boldsymbol{D}$, Thresholded BOLD ( $\boldsymbol{B}$ ) and CBV (D) fMRI activation group maps for each stimulus. Odor stimulation (green) evoked the greatest bilateral responses in superficial layers for both BOLD and CBV fMRI. LOT stimulation (red) produced a unilateral response in superficial and middle layers for BOLD and middle layers only for CBV fMRI, the latter showing a characteristic thin, ring-like pattern especially in posterior slices. AC stimulation (blue) induced a weak BOLD response, whereas CBV fMRI produced a strong bilateral response in deep layers. When the three stimulus maps were combined, the BOLD fMRI activation patterns were irregular and were poorly localized to the neural-specific layers, whereas CBV maps were layer specific and clearly segregated. These group images were calculated using a within-subject one-way ANOVA second-level analysis in SPM8: six rats (one rat removed for BOLD LOT stimulation only due to inconsistent stimulus delivery), voxelwise threshold of $p<0.01$ with a minimum number of clustered active voxels equal to 20 for a familywise error correction of $p<0.01,9$ (BOLD) and 10 (CBV) degrees of freedom. For BOLD AC stimulation only, the threshold was reduced to display better the relatively weaker activations: voxelwise and familywise threshold of $p<0.05$ (minimum number of clustered active voxels equal to 56). The black or white outlines represent the inner and outer boundaries of $\mathrm{GL}$ and the outer boundary of MCL. The anatomical underlay is the average of the coregistered anatomical images and the functional images display absolute $t$-values to preserve the red-green-blue depictions of the three stimuli and because nearly all of the activity at this threshold was positive (Fig. 4). Slices with incomplete coverage from all rats were removed from the display. In the most posterior bulb slice, example line profiles (from the white rectangles in $\boldsymbol{B}$ and $\boldsymbol{D}, 330 \mu \mathrm{m}$ thick) showed broad activation peaks for each stimulus in BOLD $(\boldsymbol{C})$ and discretely separated laminar activation patterns in CBV $(\boldsymbol{E})$ fMRI without a statistical threshold (mean \pm SD). Refer to the ROl analyses of Figure 7 for laminar activation patterns independent of the line position chosen. Vertical black bar, midline; vertical yellow bars, approximate locations of medial and lateral EPL in each bulb.

change across all layers for each individual stimulus and bulb (Fig. 7A). Odor stimulation evoked peak BOLD responses in the neural-specific GL; however, the responses to LOT and AC stimulations were also largest in this layer even though the predicted synaptic activity is located in the middle and deep layers, respectively. These profiles were opposite to the baseline SNR profile across layers, with SNR increasing from superficial to deep bulb layers, precluding that these functional observations can be ascribed to SNR deficiencies. The normalized signal changes were not significantly different for the 3 stimuli at all 7 layers (single-factor ANOVA at each of the 7 ROIs, each test had $p>$ $0.01, \mathrm{df}=2,26)$.
CBV fMRI responses are distinctly localized to the layerspecific neuronal activity

Contrary to the BOLD fMRI responses, we observed CBV responses that were highly localized to the layer-specific neuronal activity for all three stimuli (Fig. 6D). For the same line profile as BOLD, the peak activations for CBV were clearly separated for all stimulus conditions. Specifically, in the CBV $t$-statistic maps, $\mathrm{CBV}$ responses peaked in the superficial bulb layers for odor, in middle layers for right-bulb LOT, and in deep layers for AC stimulations (Fig. 6E). We observed similar laminar patterns for the ROI analyses without a statistical threshold: signals peaked in the bilateral ONL and GL for odor stimulation, in unilateral right 
A

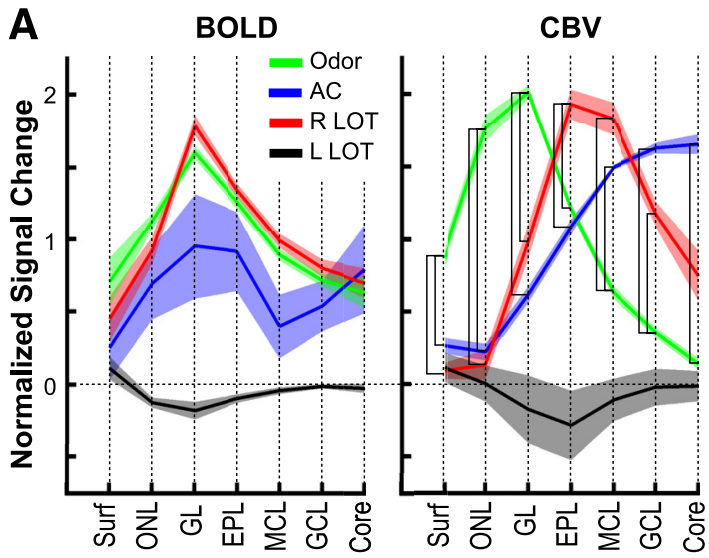

B

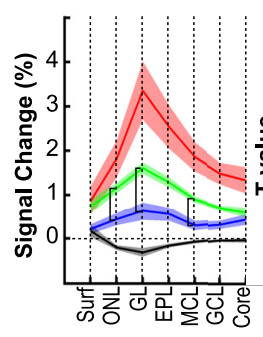

C

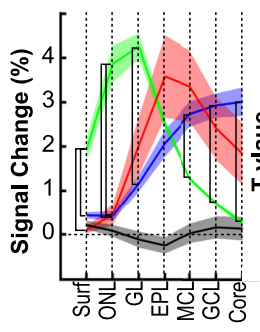

BOLD

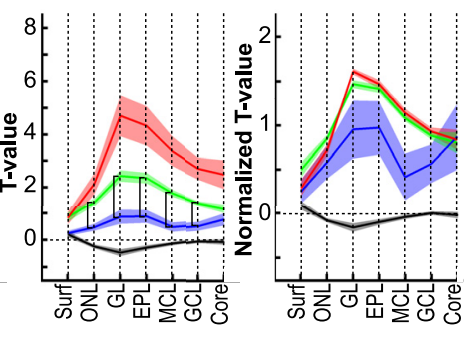

CBV

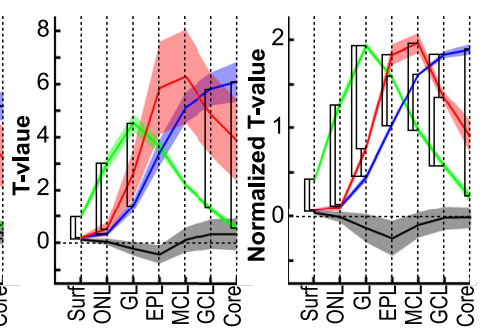

Figure 7. ROl analyses of raw and normalized fMRI responses show a greater specificity of CBV responses to the layers of increased neural activity compared with BOLD. ROl analyses show the relative laminar activations for each stimulus (mean $\pm S E M$ ). The mean activations between stimuli were compared at each layer with one-way ANOVA and two-sample $t$ tests (black brackets represent significance, $p<0.001$, uncorrected). No thresholds were applied in the ROI analyses. Because almost no activation is expected in the left bulb during right LOT stimulation, the left and right bulbs were reported separately and, when applicable, the activity in the left bulb was normalized by that of the right. $\boldsymbol{A}$, Mean fMRI signal change at each bulb layer was normalized by the average absolute response across all seven layers to control for variation in the $\mathrm{FMRI}$ response across stimuli and bulbs. For BOLD, the odor (green, $n=12$ bulbs), LOT (red, $n=5$ right bulbs), and AC (blue, $n=12$ bulbs) laminar patterns peak in the superficial GL. On the contrary, the CBV laminar patterns peak in the same layers as the directly evoked neuronal activity for all three stimulus conditions. Specifically, the CBV response to odor (green, $n=12$ bulbs) peaks in superficial (ONL and GL), right LOT (red, $n=6$ right bulbs) in right middle EPL and MCL layers and AC (blue, $n=12$ bulbs) in deep layers (GCL and core). Unexpectedly, small contralateral (left) bulb decreases in BOLD (black, $n=5$ left bulbs) and CBV (black, $n=6$ left bulbs) responses were observed during LOT stimulation. $\boldsymbol{B}, \boldsymbol{C}, \mathrm{BOLD}(\boldsymbol{B})$ and $C B V(\boldsymbol{C})$ fMRI for the mean signal change without normalization and the mean $t$-values with and without normalization, respectively, show similar laminar profiles as $\boldsymbol{A}$ and supports that our observations are independent of the ROI analysis method used. Surf, Bulb surface.

EPL and right MCL for LOT stimulation, and in bilateral GCL and core for AC stimulation (Fig. 7A,C). The normalized signal changes were significantly different for the 3 stimuli at all 7 layers ( single-factor ANOVA at each ROI, each test had $p<0.001, \mathrm{df}=$ 2,27 ) and post hoc 2-sample $t$ tests (Fig. $7 A, p<0.001$ at black brackets between stimuli). Note that the CBV $t$-values, a contrastto-noise (CNR) equivalent, are generally greater for odor and $\mathrm{AC}$

(but not LOT) stimulations compared with BOLD (Fig. $7 B, C$, middle).

Unexpectedly, during right LOT stimulation, weak negative BOLD and CBV fMRI changes were sometimes observed (3 of 6 rats) in the contralateral (left) bulb. For CBV, the negativity peaked in left EPL $(-0.3 \pm 0.2 \%)$ as shown in the no-threshold ROI analyses (Fig. 7C, left). This negative change was $\sim 10$ times smaller than the positive fMRI response in ipsilateral (right) EPL $(3.6 \pm 0.9 \%)$. The exact mechanism of this change is currently unknown, but could provide important clues to how different neural processes couple to the hemodynamic response.

\section{Layer- and stimulus-specific hemodynamic time courses explain some discrepancies between the BOLD and CBV fMRI layer specificities}

To examine the temporal dynamics of these vascular responses, the average stimulus-dependent time courses of all voxels in the right bulb (no threshold, layer independent) were determined for BOLD and CBV fMRI and compared with the CBF responses at the dorsal surface of the bulb using LDF [downsampled to $2 \mathrm{~s}$ (TR) from Fig. 3, black lines in middle row] and mean MUA changes at MCL (downsampled to $2 \mathrm{~s}$ from Fig. 3, black lines in bottom row) evoked by odor, LOT, and AC stimulations in five separate rats (Fig. 8A). All BOLD, CBV, and CBF responses immediately increased after stimulation onset regardless of the stimulation pathway. However, apparent discrepancies exist after that: time to peak, the presence of a plateau, decay time, and the presence of a poststimulus undershoot. Of particular interest is the striking difference between AC and LOT stimulation-evoked responses despite the activation of the same inhibitory granule cells. Hemodynamic responses evoked by AC stimulation reached a peak almost immediately after stimulation onset: time to $90 \%$ of the maximal peak for BOLD $=6.0 \pm 0.4 \mathrm{~s}(n=6 \mathrm{right}$ bulbs $), \mathrm{CBV}=13.7 \pm 8.7 \mathrm{~s}(n=6)$, and $\mathrm{CBF}=10.9 \pm 3.8 \mathrm{~s}(n=$ $5)$. Conversely, for LOT stimulation, after the termination of an initial brief response, the amplitude of the signal gradually increased until stimulation offset: time to $90 \%$ of the maximal peak for $\mathrm{BOLD}=33.4 \pm 9.3 \mathrm{~s}(n=5), \mathrm{CBV}=44.3 \pm 9.0 \mathrm{~s}(n=6)$, and $\mathrm{CBF}=50.4 \pm 6.5 \mathrm{~s}(n=5)$. To determine whether the unusual fMRI responses of LOT stimulation are induced by nonspecific global effects, we measured MABP changes during fMRI studies. LOT stimulation induced small, but noticeable MABP changes in three of six rats, likely due to the increased $-200 \mu \mathrm{A}$ amplitude and $200 \mu$ s pulse width for electric stimulations compared with $\mathrm{CBF}$ studies $(-150 \mu \mathrm{A}, 100 \mu \mathrm{s})$. The average MABP change observed during LOT stimulation for the pooled BOLD and CBV trials peaked at $10 \mathrm{~s}(-3.3 \pm 3.9 \%$, mean $\pm \mathrm{SD}$ ) and returned to baseline $\sim 20 \mathrm{~s}$ after stimulation onset, which approximately corresponds to the signal return to baseline after the initial peak. However, these peculiar fMRI responses evoked by LOT stimulation cannot be a mere product of this small blood pressure change because of their similarity to the $\mathrm{CBF}$ time courses, where MABP changes were negligible (Fig. 3). Furthermore, time courses of MUA changes caused by LOT and AC stimulations, which are indicative of the excitation of inhibitory granule cells, had varying degrees of similarity to the hemodynamic responses (Fig. 8A). In this figure, mitral cell spiking was recorded to monitor the stimulation effects on total circuit activity. For LOT and AC stimulations, the mitral cell spiking activity was inverted (indicated by the plus and minus signs) to show that inhibitory neurons increased their activity during stimulation. Hemodynamic responses were moderately correlated $\left(r^{2}\right.$, Pearson's 
A

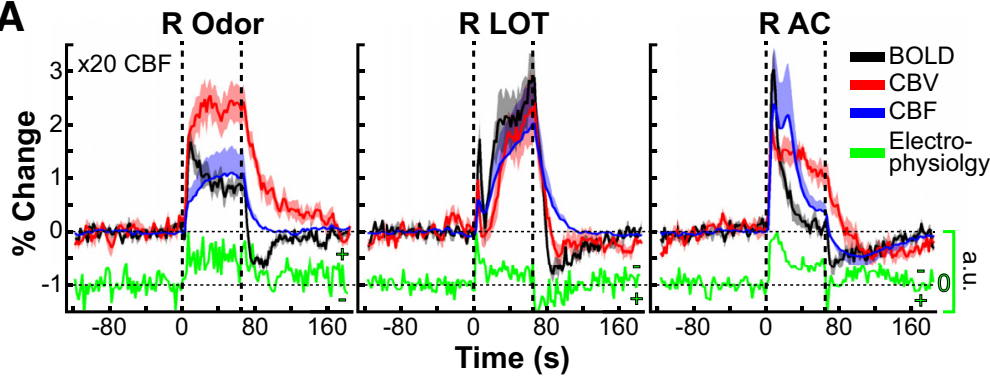

B
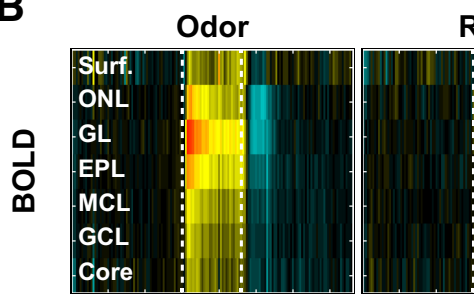

R LOT
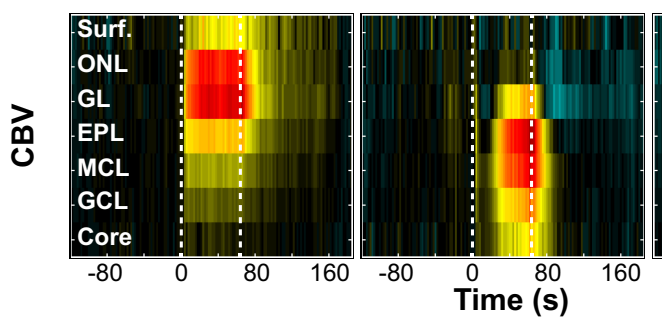

C
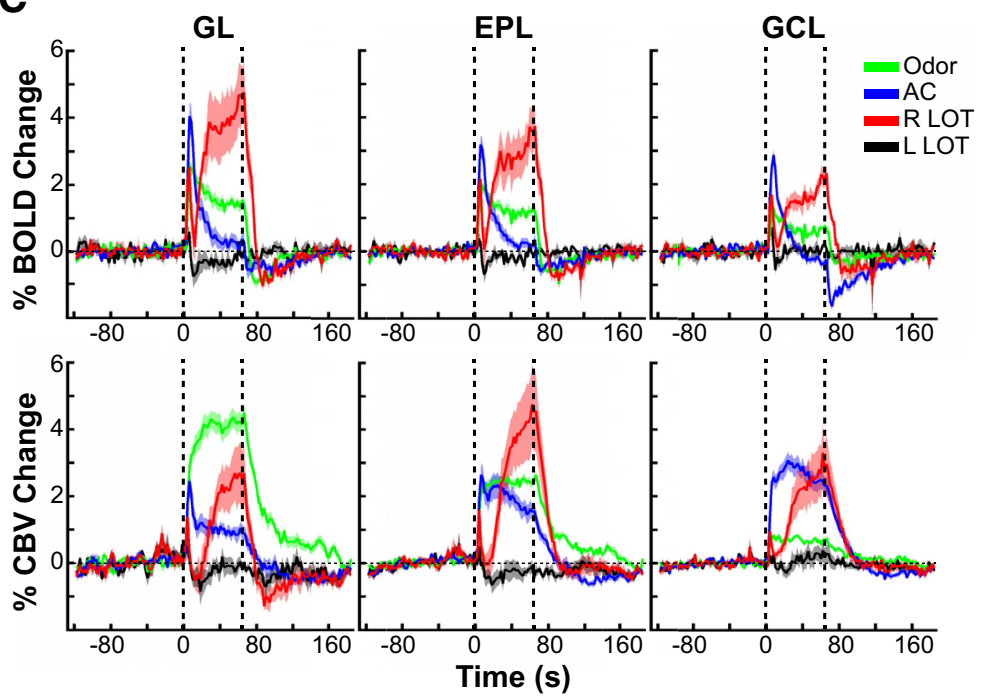

Figure 8. Group layer-specific hemodynamic time courses are stimulus-dependent and provide insight on neurovascular mechanisms. $A$, Time courses (mean \pm SEM) for BOLD (black, $n=6$ right bulbs for odor and AC, $n=5$ for LOT) and CBV (red, $n=6$ for all three stimuli) fMRI for all voxels in the right bulb (layer independent) and CBF (blue, $n=5$ different rats, downsampled from the black lines in the middle row of Fig. 3) measured on the right bulb surface using LDF show increases in BOLD, CBV, and CBF responses during odor ( $5 \%$ amyl acetate in mineral oil) and electrical stimulations of $\mathrm{L} 0 \mathrm{~T}$ and $\mathrm{AC}[-200(-150) \mu \mathrm{A}, 200(100) \mu \mathrm{s}$ pulse width for $\mathrm{fMRI}(\mathrm{CBF}), 40 \mathrm{~Hz}, 64$ s duration]. In addition, changes in spike discharge rates at $\mathrm{MCL}$ (green, $n=5$ same rats as the (BF measurements, downsampled from the black lines in the bottom row of Fig. 3) were shown here as a simple indicator of the relative neural circuit activity because the mitral cell is the common output of all three stimulation pathways. An increase in inhibitory neural activation is inferred from a decrease in MCL spiking during LOT and AC stimulations (Fig. 3), so the sign of these electrophysiological time courses was inverted (as indicated by the negative sign above the baseline). The electrophysiological time courses have similar temporal shapes as CBF and CBV for odor and CBF and BOLD for AC stimulations, but the hemodynamic measures are dissimilar for LOT stimulation except for the initial transient response at stimulation onset. For clarity, only one side of the error ranges was plotted for CBF and no error ranges were plotted for the electrophysiology in arbitrary units (a.u.) with the baseline shifted from the hemodynamic measurements to clearly show the relative shape of the circuit activity over time. The vertical dashed lines represent the stimulus onset and offset. $B$, ROI analysis was extended to calculate the average BOLD and CBV fMRI time courses for each layer during all 3 stimulations and displayed as a 2D color plot. C, In addition, traditional BOLD and CBV time courses were plotted for the specific layers of preferentially evoked synaptic activity (GL for odor, EPL for LOT, and GCL for AC). Time courses due to odor stimulation ( $n=12$ bulbs) have typical BOLD and CBV fMRI responses that are both greatest in the linear correlation, $\left.{ }^{*} p<0.001\right)$ to the MUA changes for odor $\left(\mathrm{CBF}=0.44^{*}\right.$, $\left.\mathrm{BOLD}=0.24^{*}, \mathrm{CBV}=0.48^{*}\right)$ and $\mathrm{AC}$ $\left(\mathrm{CBF}=0.44^{*}, \mathrm{BOLD}=0.39^{*}, \mathrm{CBV}=\right.$ $\left.0.36^{*}\right)$ stimulations, whereas correlations were poor for LOT $(\mathrm{CBF}=0.01$, $\left.\mathrm{BOLD}=0.09^{*}, \mathrm{CBV}=0.02\right)$. It is noted that MUAs were recorded from a single point, unlike the hemodynamic measures, and different rats were used for fMRI and MUA experiments.

We then examined the BOLD and CBV fMRI time courses at each layer (Fig. $8 B, C)$. We used the same methods as the previous ROI analyses without a threshold applied, whereby the time courses for all voxels in each ROI were averaged within each bulb before being averaged across bulbs. First, although the greatest positive BOLD signal changes were located to GL for all three stimuli, the poststimulus undershoot was better localized to the layers of evoked synaptic activity, especially for odor ( $n=12$ bulbs) and AC $(n=12)$. In these two cases, the positive $\mathrm{CBF}$ responses diminished faster than the prolonged CBV responses and likely contributed to the negative BOLD changes (Fig. 8A-C). Second, small BOLD and CBV increases were observed immediately after the start of stimulation in the contralateral (left) bulb during right LOT stimulation, which may be caused by systemic MABP changes and requires further investigation to determine its origins. However, the magnitude of this change is much smaller than that of the ipsilateral (right) EPL (Fig. 8C, middle). In addition, the initial return to baseline in the first $\sim 10$ s of LOT stimulation has slightly dif-

\footnotetext{
neural-specific GL. After the offset of odor stimulation, a fast CBF return, a slow CBV return, and a prominent BOLD undershoot were observed in $\mathrm{GL}$ and surrounding layers. During right LOT stimulation, small biphasic $\mathrm{FMRI}$ responses are observed in the contralateral (left) bulb ( $n=5$ for BOLD, $n=6$ for (BV). The right bulb $L 0 T$ time courses for all three hemodynamic measures show a fast initial peak that returns to baseline before slowly increasing to a second peak near the stimulus termination. More interestingly, the slow time to the second peak is unlike odor and AC stimulations and may indicate a unique neuro-vascular coupling to the evoked dendrodendritic synapses. Interestingly, for AC stimulation, the BOLD and $C B F$ time courses are similar, but notably different from $C B V$, suggesting that $C B F$ and $C B V$ may be regulated by different mechanisms. In BOLD and CBV ( $n=12$ bulbs), there is an initial fast, positive response that spans across all bulb layers. Subsequently, BOLD returns to baseline during stimulation and has a neural-specific poststimulus undershoot in deep layers, whereas the CBV response remains near the plateau in deep layers but decreases in GL. The poststimulus BOLD undershoot and the later CBV response are more specific than the positive BOLD and early CBV responses, respectively.
} 
ferent onset times and temporal widths across the different layers and fMRI contrasts in the right bulb (Fig. $8 B$, middle), suggesting a local, neuronal source rather than a systemic one. Finally, for AC stimulation (Fig. $8 A-C$ ), the BOLD response initially increased nonspecifically across all of the bulb layers and returned nearly to baseline before the stimulation ceased, which was generally similar to the CBF response. This quick return to baseline is the likely cause of the poor sensitivity of the AC fMRI maps (Figs. $4,6,7)$ because this time course shape will correlate poorly with the predicted HRF of the general linear model in SPM8. For CBV, changes soon after the onset of stimulation were similar across all layers, whereas the later $\mathrm{CBV}$ responses were specific to the deep layers. This indicates that a later CBV fMRI response is more specific to the active neuronal sites than at an earlier time (Berwick et al., 2008; Moon et al., 2013).

\section{Discussion}

The main purpose of our study was to test whether the blood supply was regulated at individual layers in the olfactory bulb. We found that the laminar localization of CBV responses was obvious and largest at the input layer for each of the stimulation pathways. Conversely, the laminar localization for BOLD responses was not easily discernible and largest in superficial bulb layers, with some exception to the poststimulus undershoot, regardless of the stimulation pathway. Therefore, our results support the hypothesis that the largest hemodynamic response, namely CBV, occurs precisely at the evoked synapse. Furthermore, the stimulus-dependent fMRI time courses provide insight into the vascular and neuronal origins of the evoked signal changes, whereas the hemodynamic increases to LOT and AC stimulations may indicate that inhibitory neuronal activities dilate vessels.

\section{CBV fMRI changes are highly layer specific, whereas BOLD is poor}

We demonstrated that the hemodynamic response, particularly $\mathrm{CBV}$, was colocalized with the site of the preferentially evoked, layer-specific neuronal activities (Figs. 6, 7, 8). The discrepancy between the layer specificities of the BOLD and CBV responses can be explained by the underlying signal sources. First, BOLD fMRI is sensitive to the absolute amount of deoxyhemoglobin in the blood, which is modulated by blood oxygenation and volume (Kim and Ogawa, 2012). During stimulation, an increase in CBF will increase oxygenation and BOLD signals, whereas an increase in blood volume will decrease BOLD signals. Therefore, the large, layer-specific CBV changes measured in the current study will reduce the BOLD changes in the same layers and thus obscure the true location of activation. Second, BOLD fMRI is weighted by the baseline total and, to a greater degree, venous blood volumes because a change in deoxyhemoglobin content is a multiple of oxygenation and volume. In the olfactory bulb, the baseline blood volumes of all vessels and just that of microvessels, including capillaries, are greatest in the superficial layers and decrease with laminar depth (Poplawsky and Kim, 2014). In fact, the BOLD ROI profiles are similarly weighted toward superficial layers for the three different stimulus pathways and thus may simply be a reflection of the baseline blood volume. In contrast, the CBV laminar profiles for all three stimulation types are specific to the layer of evoked neuronal activity. The single fact that AC stimulation evokes the greatest CBV change in deep bulb layers, where there is the least baseline blood volume, suggests that microvessels are locally regulated. Third, BOLD fMRI, but not CBV, has an increased sensitivity to nonspecific draining veins and to intra- vascular inflow effects in large vessels. Finally, CBV fMRI generally has a higher CNR than BOLD in cortical tissue, but less CNR in large vessels due to baseline signal reductions caused by the contrast agent (Mandeville et al., 2004). Therefore, CBV fMRI is preferable for high-resolution animal studies and may be used to map neighboring microcircuit activity, including laminar processing.

The exact underlying mechanism of microvessel regulation is currently unclear, but arterioles controlled by astrocytes (Zonta et al., 2003; Mulligan and MacVicar, 2004) and capillaries controlled by pericytes (Peppiatt et al., 2006) have lately drawn considerable attention, although their significance to in vivo systems is highly controversial (Nizar et al., 2013; Hall et al., 2014; Hill et al., 2015; Otsu et al., 2015). In addition, vasodilation propagated by the vascular endothelium (Hillman, 2014) or direct control of microvessels innervated by inhibitory neurons (Cauli et al., 2004) may also play a role.

\section{Temporal dynamics of the layer-specific fMRI responses}

The time-dependent hemodynamic changes provide further insight into how the vessels respond to the evoked neuronal activity. First, different vessels have different dynamic properties when activated; larger, "upstream" arterioles respond faster and more transiently compared with the more localized, "downstream" microvasculature (Berwick et al., 2008; Moon et al., 2013). Large and transient hemodynamic responses are observed immediately after LOT and AC stimulation onset (Fig. 8B,C), most evidently in the BOLD AC time courses, where the initial response spans nonspecifically across all laminar depths and is indicative of penetrating arterioles. However, the later, localized CBV responses are much slower than the BOLD and CBF responses for all stimuli, suggesting microvessel dilation. Second, each stimulus produces fMRI time courses that are similar in shape across all layers, but are different between stimuli (Fig. 8C). This is of particular importance because the vascular compositions are the same within each ROI and precludes the possibility that different vascular reactivities in different layers cause the different fMRI responses. A neuronal origin is further supported by the limited correlation of the hemodynamic and electrophysiological measures (Fig. 8A). Third, a localized poststimulus BOLD undershoot was observed for AC and odor stimulations (Fig. 8). This undershoot can be due to sustained CBV increases, prolonged oxygen extraction from the blood, and/or to CBF decreases (Mandeville et al., 1998; Chen and Pike, 2009; Hua et al., 2011). Regardless of its exact origins, the poststimulus BOLD changes are better localized to the active layers than the positive BOLD, especially for AC stimulation.

The most unexpected observation is that different hemodynamic time courses were elicited from LOT and AC stimulations despite the same population of granule cells being activated. Although the exact reasons for these differences are unclear, they likely originate from the synapse, so several speculations are deserved. First, the slowly increasing hemodynamic response evoked by LOT may be explained by a greater control of the vasculature by astrocytes (Attwell et al., 2010) because Schulz et al. (2012) demonstrated that time courses of slow glial responses were able to predict prolonged fMRI responses. Alternatively, the slow and fast hemodynamic responses evoked by LOT and AC stimulations may reflect the slow synaptic kinetics observed in the distal dendrites (in EPL) of granule cells and the fast kinetics in proximal dendrites (in GCL), respectively (Balu and Strowbridge, 2007). NMDA receptors, which have slower kinetics than AMPA receptors, have a different and more dominant role in 
granule cell activation at the dendrodendritic synapses in EPL (Isaacson and Strowbridge, 1998; Schoppa et al., 1998; Chen et al., 2000) and may be crucial for the LOT-stimulation-evoked hemodynamic response. Further, this response may be modulated by the activation of metabotropic glutamate receptors, which are highly expressed on the dendrodendritic synapses in EPL and increase the granule cells' excitability on the order of minutes (Dong et al., 2007).

\section{Neurovascular coupling to glutamatergic neurons}

We observed the largest CBV increases in GL for odor stimulation, where we primarily evoked the apical dendrites of excitatory mitral cells. However, it is debatable whether postsynaptic activities are responsible for these odor-evoked hemodynamic responses. Exclusive presynaptic contributions were reported by Gurden et al. (2006) and Petzold et al. (2008), who showed that such responses were insensitive to the application of postsynaptic ionotropic glutamate receptor (AMPA and NMDA) antagonists, but were significantly suppressed by an astrocytic glutamate transporter blocker. On the contrary, Chaigneau et al. (2007) found that odor-evoked CBF responses were not affected by local injections of AMPA and NMDA receptor antagonists into a single glomerulus, but were significantly suppressed by injecting the antagonists beyond the single glomerulus. Therefore, they concluded that hemodynamic responses are regulated by the cooperation of several glomeruli, particularly with higher odor concentrations, and that postsynaptic neuronal activation is necessary to elicit hemodynamic responses. In addition, glomerular astrocyte transporters are activated by glutamate release from both the presynaptic olfactory nerve terminals and secondary dendrites of the postsynaptic mitral cells after spike discharges (De Saint Jan and Westbrook, 2005). These studies collectively suggest that both astrocytic and postsynaptic neuronal activities play a role in odor-evoked neurovascular coupling.

\section{Neurovascular coupling to GABAergic neurons}

We observed the largest CBV increases in EPL for LOT and in GCL for AC stimulations, where we primarily evoked inhibitory granule cells, confirmed by LFPs (Fig. $2 A, B$ ) and mitral cell spiking suppressions (Fig. 2C,D). These layer-specific results suggest that the vascular response is regulated at the primary synapse and may be partially controlled by the postsynaptic activities of inhibitory neurons, assuming that these neurons couple similarly to the vasculature as the odor-evoked mitral cells (described previously). Furthermore, direct photoactivation of cortical inhibitory neurons expressing the light-gated cation channel channelrhodopsin-2 (ChR2) suggests that glutamatergic transmission is not required to evoke hemodynamic responses (Anenberg et al., 2015). However, the mechanism of neurovascular coupling in this ChR2-expressing inhibitory mouse model may be different from postsynaptically activated GABAergic neurons because ChR2 is a nonspecific cation channel that is permeable to $\mathrm{Na}^{+}, \mathrm{K}^{+}$, and $\mathrm{Ca}^{2+}$. In addition to glutamatergic transmission, the role of concomitant GABA release on our observed hemodynamic responses is unknown, but we can speculate that GABA release is less essential because we did not observe peak fMRI responses in EPL for AC stimulation (Fig. 1A). Furthermore, interneurons are known to corelease peptides that dilate or constrict vessels (Cauli et al., 2004), although we observed only net increases in the hemodynamic responses evoked by inhibitory pathways. Further pharmacological studies by blocking or modulating specific receptors are necessary to test these outstanding issues directly.

\section{References}

Anenberg E, Chan AW, Xie Y, LeDue JM, Murphy TH (2015) Optogenetic stimulation of GABA neurons can decrease local neuronal activity while increasing cortical blood flow. J Cereb Blood Flow Metab. In press.

Attwell D, Laughlin SB (2001) An energy budget for signaling in the grey matter of the brain. J Cereb Blood Flow Metab 21:1133-1145. Medline

Attwell D, Buchan AM, Charpak S, Lauritzen M, Macvicar BA, Newman EA (2010) Glial and neuronal control of brain blood flow. Nature 468: 232-243. CrossRef Medline

Balu R, Strowbridge BW (2007) Opposing inward and outward conductances regulate rebound discharges in olfactory mitral cells. J Neurophysiol 97:1959-1968. CrossRef Medline

Balu R, Pressler RT, Strowbridge BW (2007) Multiple modes of synaptic excitation of olfactory bulb granule cells. J Neurosci 27:5621-5632. CrossRef Medline

Berwick J, Johnston D, Jones M, Martindale J, Martin C, Kennerley AJ, Redgrave P, Mayhew JE (2008) Fine detail of neurovascular coupling revealed by spatiotemporal analysis of the hemodynamic response to single whisker stimulation in rat barrel cortex. J Neurophysiol 99:787-798. CrossRef Medline

Borowsky IW, Collins RC (1989) Metabolic anatomy of brain: A comparison of regional capillary density, glucose metabolism, and enzyme activities. J Comp Neurol 288:401-413. CrossRef Medline

Boyd AM, Sturgill JF, Poo C, Isaacson JS (2012) Cortical feedback control of olfactory bulb circuits. Neuron 76:1161-1174. CrossRef Medline

Cauli B, Tong XK, Rancillac A, Serluca N, Lambolez B, Rossier J, Hamel E (2004) Cortical GABA interneurons in neurovascular coupling: relays for subcortical vasoactive pathways. J Neurosci 24:8940-8949. CrossRef Medline

Chaigneau E, Tiret P, Lecoq J, Ducros M, Knöpfel T, Charpak S (2007) The relationship between blood flow and neuronal activity in the rodent olfactory bulb. J Neurosci 27:6452-6460. CrossRef Medline

Chen G, Wang F, Gore JC, Roe AW (2013) Layer-specific BOLD activation in awake monkey V1 revealed by ultra-high spatial resolution functional magnetic resonance imaging. Neuroimage 64:147-155. CrossRef Medline

Chen JJ, Pike GB (2009) Origins of the BOLD post-stimulus undershoot. Neuroimage 46:559-568. CrossRef Medline

Chen WR, Xiong W, Shepherd GM (2000) Analysis of relations between NMDA receptors and GABA release at olfactory bulb reciprocal synapses. Neuron 25:625-633. CrossRef Medline

De Saint Jan D, Westbrook GL (2005) Detecting activity in olfactory bulb glomeruli with astrocyte recording. J Neurosci 25:2917-2924. CrossRef Medline

Dong HW, Hayar A, Ennis M (2007) Activation of group i metabotropic glutamate receptors on main olfactory bulb granule cells and periglomerular cells enhances synaptic inhibition of mitral cells. J Neurosci 27: 5654-5663. CrossRef Medline

Goense J, Merkle H, Logothetis NK (2012) High-resolution fMRI reveals laminar differences in neurovascular coupling between positive and negative BOLD responses. Neuron 76:629-639. CrossRef Medline

Gurden H, Uchida N, Mainen ZF (2006) Sensory-evoked intrinsic optical signals in the olfactory bulb are coupled to glutamate release and uptake. Neuron 52:335-345. CrossRef Medline

Hall CN, Reynell C, Gesslein B, Hamilton NB, Mishra A, Sutherland BA, O'Farrell FM, Buchan AM, Lauritzen M, Attwell D (2014) Capillary pericytes regulate cerebral blood flow in health and disease. Nature 508: 55-60. CrossRef Medline

Harel N, Lin J, Moeller S, Ugurbil K, Yacoub E (2006) Combined imaginghistological study of cortical laminar specificity of fMRI signals. Neuroimage 29:879-887. CrossRef Medline

Harris JJ, Jolivet R, Attwell D (2012) Synaptic energy use and supply. Neuron 75:762-777. CrossRef Medline

Hill RA, Tong L, Yuan P, Murikinati S, Gupta S, Grutzendler J (2015) Regional blood flow in the normal and ischemic brain is controlled by arteriolar smooth muscle cell contractility and not by capillary pericytes. Neuron 87:95-110. CrossRef Medline

Hillman EM (2014) Coupling mechanism and significance of the BOLD signal: a status report. Annu Rev Neurosci 37:161-181. CrossRef Medline

Hua J, Stevens RD, Huang AJ, Pekar JJ, van Zijl PC (2011) Physiological origin for the BOLD poststimulus undershoot in human brain: vascular compliance versus oxygen metabolism. J Cereb Blood Flow Metab 31: 1599-1611. CrossRef Medline 
Isaacson JS, Strowbridge BW (1998) Olfactory reciprocal synapses: dendritic signaling in the CNS. Neuron 20:749-761. CrossRef Medline

Kim SG, Ogawa S (2012) Biophysical and physiological origins of blood oxygenation level-dependent fMRI signals. J Cereb Blood Flow Metab 32:1188-1206. CrossRef Medline

Koopmans PJ, Barth M, Norris DG (2010) Layer-specific BOLD activation in human V1. Hum Brain Mapp 31:1297-1304. CrossRef Medline

Korol DL, Brunjes PC (1992) Unilateral naris closure and vascular development in the rat olfactory bulb. Neuroscience 46:631-641. CrossRef Medline

Laaris N, Puche A, Ennis M (2007) Complementary postsynaptic activity patterns elicited in olfactory bulb by stimulation of mitral/tufted and centrifugal fiber inputs to granule cells. J Neurophysiol 97:296-306. CrossRef Medline

Lecoq J, Tiret P, Najac M, Shepherd GM, Greer CA, Charpak S (2009) Odorevoked oxygen consumption by action potential and synaptic transmission in the olfactory bulb. J Neurosci 29:1424-1433. CrossRef Medline

Mandeville JB, Marota JJ, Kosofsky BE, Keltner JR, Weissleder R, Rosen BR, Weisskoff RM (1998) Dynamic functional imaging of relative cerebral blood volume during rat forepaw stimulation. Magn Reson Med 39:615624. CrossRef Medline

Mandeville JB, Jenkins BG, Chen YC, Choi JK, Kim YR, Belen D, Liu C, Kosofsky BE, Marota JJ (2004) Exogenous contrast agent improves sensitivity of gradient-echo functional magnetic resonance imaging at $9.4 \mathrm{~T}$. Magn Reson Med 52:1272-1281. CrossRef Medline

Markopoulos F, Rokni D, Gire DH, Murthy VN (2012) Functional properties of cortical feedback projections to the olfactory bulb. Neuron 76: 1175-1188. CrossRef Medline

Moon CH, Fukuda M, Kim SG (2013) Spatiotemporal characteristics and vascular sources of neural-specific and -nonspecific fMRI signals at submillimeter columnar resolution. Neuroimage 64:91-103. CrossRef Medline

Mori K, Takagi SF (1978) An intracellular study of dendrodendritic inhibitory synapses on mitral cells in the rabbit olfactory bulb. J Physiol 279: 569-588. CrossRef Medline

Mulligan SJ, MacVicar BA (2004) Calcium transients in astrocyte endfeet cause cerebrovascular constrictions. Nature 431:195-199. CrossRef Medline

Nakashima M, Mori K, Takagi SF (1978) Centrifugal influence on olfactory bulb activity in the rabbit. Brain Res 154:301-316. CrossRef Medline

Nickell WT, Shipley MT (1988) Neurophysiology of magnocellular forebrain inputs to the olfactory bulb in the rat: frequency potentiation of field potentials and inhibition of output neurons. J Neurosci 8:44924502. Medline

Nickell WT, Shipley MT (1993) Evidence for presynaptic inhibition of the olfactory commissural pathway by cholinergic agonists and stimulation of the nucleus of the diagonal band. J Neurosci 13:650-659. Medline

Nizar K, Uhlirova H, Tian P, Saisan PA, Cheng Q, Reznichenko L, Weldy KL, Steed TC, Sridhar VB, MacDonald CL, Cui J, Gratiy SL, Sakadzić S, Boas DA, Beka TI, Einevoll GT, Chen J, Masliah E, Dale AM, Silva GA, et al. (2013) In vivo stimulus-induced vasodilation occurs without IP3 receptor activation and may precede astrocytic calcium increase. J Neurosci 33:8411-8422. CrossRef Medline

Olman CA, Harel N, Feinberg DA, He S, Zhang P, Ugurbil K, Yacoub E (2012) Layer-specific fMRI reflects different neuronal computations at different depths in human V1. PLoS One 7:e32536. CrossRef Medline

Oswald AM, Urban NN (2012) There and back again: the corticobulbar loop. Neuron 76:1045-1047. CrossRef Medline

Otsu Y, Couchman K, Lyons DG, Collot M, Agarwal A, Mallet JM, Pfrieger
FW, Bergles DE, Charpak S (2015) Calcium dynamics in astrocyte processes during neurovascular coupling. Nat Neurosci 18:210-218. Medline

Peppiatt CM, Howarth C, Mobbs P, Attwell D (2006) Bidirectional control of CNS capillary diameter by pericytes. Nature 443:700-704. CrossRef Medline

Petzold GC, Albeanu DF, Sato TF, Murthy VN (2008) Coupling of neural activity to blood flow in olfactory glomeruli is mediated by astrocytic pathways. Neuron 58:897-910. CrossRef Medline

Polimeni JR, Fischl B, Greve DN, Wald LL (2010) Laminar analysis of 7T BOLD using an imposed spatial activation pattern in human V1. Neuroimage 52:1334-1346. CrossRef Medline

Poplawsky AJ, Kim SG (2014) Layer-dependent BOLD and CBV-weighted fMRI responses in the rat olfactory bulb. Neuroimage 91:237-251. CrossRef Medline

Rall W, Shepherd GM (1968) Theoretical reconstruction of field potentials and dendrodendritic synaptic interactions in olfactory bulb. J Neurophysiol 31:884-915. Medline

Ress D, Glover GH, Liu J, Wandell B (2007) Laminar profiles of functional activity in the human brain. Neuroimage 34:74-84. CrossRef Medline

Schoppa NE, Kinzie JM, Sahara Y, Segerson TP, Westbrook GL (1998) Dendrodendritic inhibition in the olfactory bulb is driven by NMDA receptors. J Neurosci 18:6790-6802. Medline

Schulz K, Sydekum E, Krueppel R, Engelbrecht CJ, Schlegel F, Schröter A, Rudin M, Helmchen F (2012) Simultaneous BOLD fMRI and fiberoptic calcium recording in rat neocortex. Nat Methods 9:597-602. CrossRef Medline

Shepherd GM (1972) Synaptic organization of the mammalian olfactory bulb. Physiol Rev 52:864-917. Medline

Silva AC, Koretsky AP, Duyn JH (2007) Functional MRI impulse response for BOLD and CBV contrast in rat somatosensory cortex. Magn Reson Med 57:1110-1118. CrossRef Medline

Storer RJ, Butler P, Hoskin KL, Goadsby PJ (1997) A simple method, using 2 -hydroxypropyl- $\beta$-cyclodextrin, of administering $\alpha$-chloralose at room temperature. J Neurosci Methods 77:49-53. CrossRef Medline

Uva L, Strowbridge BW, de Curtis M (2006) Olfactory bulb networks revealed by lateral olfactory tract stimulation in the in vitro isolated guineapig brain. Neuroscience 142:567-577. CrossRef Medline

Vazquez AL, Fukuda M, Crowley JC, Kim SG (2014) Neural and hemodynamic responses elicited by forelimb- and photo-stimulation in channelrhodopsin-2 mice: insights into the hemodynamic point spread function. Cereb Cortex 24:2908-2919. CrossRef Medline

Xu F, Kida I, Hyder F, Shulman RG (2000) Assessment and discrimination of odor stimuli in rat olfactory bulb by dynamic functional MRI. Proc Natl Acad Sci U S A 97:10601-10606. CrossRef Medline

Xu F, Liu N, Kida I, Rothman DL, Hyder F, Shepherd GM (2003) Odor maps of aldehydes and esters revealed by functional MRI in the glomerular layer of the mouse olfactory bulb. Proc Natl Acad Sci U S A 100: 11029-11034. CrossRef Medline

Yen CC-C (2011) Cortical layer-dependent hemodynamic regulation investigated by functional magnetic resonance imaging. Doctoral dissertation, University of Pittsburgh.

Zhao F, Wang P, Hendrich K, Ugurbil K, Kim SG (2006) Cortical layerdependent BOLD and CBV responses measured by spin-echo and gradient-echo fMRI: Insights into hemodynamic regulation. Neuroimage 30:1149-1160. CrossRef Medline

Zong X, Lee J, John Poplawsky A, Kim SG, Ye JC (2014) Compressed sensing fMRI using gradient-recalled echo and EPI sequences. Neuroimage 92:312-321. CrossRef Medline

Zonta M, Angulo MC, Gobbo S, Rosengarten B, Hossmann KA, Pozzan T, Carmignoto G (2003) Neuron-to-astrocyte signaling is central to the dynamic control of brain microcirculation. Nat Neurosci 6:43-50. Medline 